\title{
Symmetry Analysis of Reversible Markov Chains
}

\author{
Stephen Boyd, Persi Diaconis, Pablo Parrilo, and Lin Xiao
}

Abstract. We show how to use subgroups of the symmetry group of a reversible Markov chain to give useful bounds on eigenvalues and their multiplicity. We supplement classical representation theoretic tools involving a group commuting with a self-adjoint operator with criteria for an eigenvector to descend to an orbit graph. As examples, we show that the Metropolis construction can dominate a max-degree construction by an arbitrary amount and that, in turn, the fastest mixing Markov chain can dominate the Metropolis construction by an arbitrary amount.

\section{Introduction}

In our work on fastest mixing Markov chains on a graph [Boyd et al. 04, Parrilo et al. 04], we encountered highly symmetric graphs with weights on the edges. Examples treated below include the graphs shown in Figures 1-5. The graphs in Figures 2, 3, 4, and 5 have weights chosen so that the stationary distribution of the associated random walk is uniform. We will show that the walk in Figure 2 mixes much more rapidly than the walk in Figure 3 and that the walk in Figure 4 mixes much more rapidly than the walk in Figure 5. For general graphs, we seek good bounds for eigenvalues and their multiplicity using available symmetry.

Let a connected graph $(V, E)$ have vertex set $V$ and undirected edge set $E$. We allow loops but not multiple edges. Let $w(e)$ be positive weights on the edges. These ingredients define a random walk on $V$ that moves from $v$ to a neighboring $v^{\prime}$ with probability proportional to $w\left(v, v^{\prime}\right)$. This walk has transition

(C) A K Peters, Ltd.

|542-795I/05 \$0.50 per page 


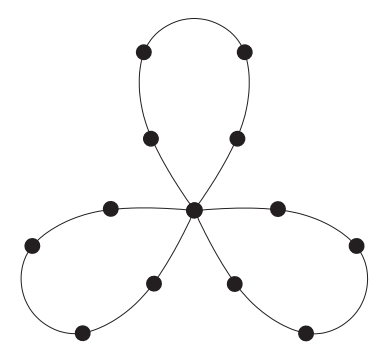

Figure I. $F_{m n}$ with $m$ petals, each a cycle of length $n$. All edges have weight 1 .

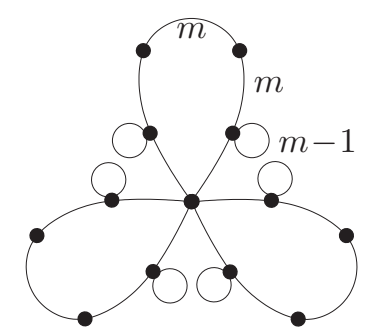

Figure 2. $F_{m n}$ with all loops having weight $m-1$, edges incident to the center having weight 1 , and other edges having weight $m$ (Metropolis weights).

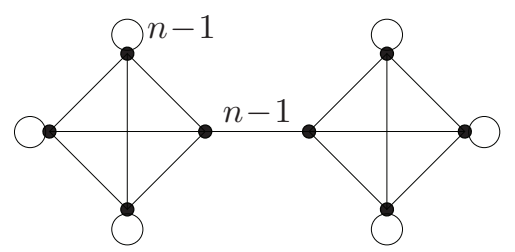

Figure 4. $K_{n}-K_{n}$ with center edge and all loops having weight $n-1$ and other edges having weight 1 .

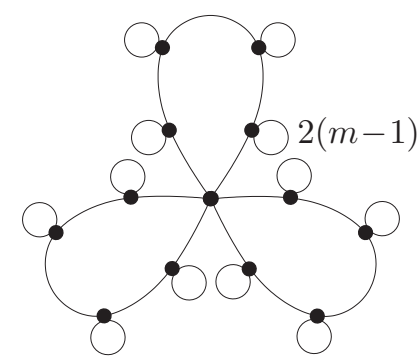

Figure 3. $F_{m n}$ with all loops having weight $2(m-1)$ and other edges having weight 1 (max-degree weights).

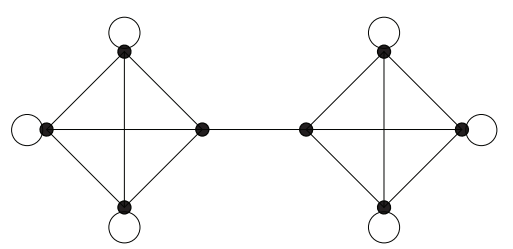

Figure 5. $K_{n}-K_{n}$ with all edges and loops having weight 1 (max-degree weights). 
matrix

$$
K\left(v, v^{\prime}\right)=\frac{w\left(v, v^{\prime}\right)}{W(v)}, \quad \text { where } \quad W(v)=\sum_{v^{\prime \prime}} w\left(v, v^{\prime \prime}\right) .
$$

The Markov chain $K$ has unique stationary distribution $\pi(v)$ proportional to the sum of the edge weights that meet at $v$ :

$$
\pi(v)=\frac{W(v)}{W}, \quad \text { where } \quad W=\sum_{v^{\prime}} W\left(v^{\prime}\right) .
$$

By inspection, the pair $K, \pi$ is reversible:

$$
\pi(v) K\left(v, v^{\prime}\right)=\pi\left(v^{\prime}\right) K\left(v^{\prime}, v\right)
$$

Reversible Markov chains are a mainstay of scientific computing through Markov chain Monte Carlo; see, e.g., [Liu 01]. Any reversible Markov chain can be represented as a random walk on an edge weighted graph. Background on reversible Markov chains can be found in the textbook of Brémaud [Brémaud 99], the lecture notes of Saloff-Coste [Saloff-Coste 97], or the treatise of Aldous and Fill [Aldous and Fill 03].

Define $L^{2}(\pi)=\{f: V \rightarrow \mathbf{R}\}$ with inner product $\left\langle f_{1}, f_{2}\right\rangle=\sum_{v} f_{1}(v) f_{2}(v) \pi(v)$. The matrix $K\left(v, v^{\prime}\right)$ operates on $L^{2}$ by

$$
K f(v)=\sum_{v^{\prime}} K\left(v, v^{\prime}\right) f\left(v^{\prime}\right)
$$

Reversibility (1.3) is equivalent to self-adjointness $\left\langle K f_{1}, f_{2}\right\rangle=\left\langle f_{1}, K f_{2}\right\rangle$. It follows that $K$ is diagonalizible with all real eigenvalues and eigenvectors.

An automorphism of a weighted graph is a permutation $g: V \rightarrow V$ such that if $\left(v, v^{\prime}\right) \in E$, then $\left(g v, g v^{\prime}\right) \in E$ and $w\left(v, v^{\prime}\right)=w\left(g v, g v^{\prime}\right)$. Let $G$ be a group of automorphisms. This group acts on $L^{2}(\pi)$ by

$$
T_{g} f(v)=f\left(g^{-1} v\right)
$$

Since $g$ is an automorphism,

$$
T_{g} K=K T_{g}, \quad \forall g \in G
$$

Proposition I.I. For random walk (1.1) on an edge weighted graph, the stationary distribution $\pi$ defined in (1.2) is invariant under all automorphisms. 
Proof.

$$
\begin{aligned}
T_{g} \pi(v) & =\frac{1}{W} \sum_{u^{\prime}} w\left(g^{-1} v, u^{\prime}\right) \\
& =\frac{1}{W} \sum_{u} w\left(g^{-1} v, g^{-1} u\right) \\
& =\frac{1}{W} \sum_{u} w(v, u)=\pi(v) .
\end{aligned}
$$

It follows that $L^{2}(\pi)$ is a unitary representation of $G$.

Example I.2. (Suggested by Robin Forman.) Let $F_{m n}$ be the graph of a "flower" with $m$ petals, each a cycle containing $n$ vertices, joined at the center vertex 0 . Thus, Figure 1 shows the flower $m=3, n=5$. If $w(e)=1$ for all $e \in E$, the stationary distribution is highly nonuniform. From (1.2),

$$
\pi(0)=\frac{1}{n}, \quad \pi(v)=\frac{1}{m n} \text { for } v \neq 0 .
$$

Our work in this area begins by considering two methods of putting weights on the edges of $F_{m n}$ to make the stationary distribution uniform. The Metropolis weights (Figure 2) turn out to lead to a more rapidly mixing chain than the max-degree weights (Figure 3). In [Boyd et al. 04, Parrilo et al. 04], we show how to find optimal weights that give the largest spectral gap. For $F_{m n}$ these improve slightly over the Metropolis weights. Our algorithms give exact numerical answers for fixed $m$ and $n$. In the present paper we give analytical results. All the algorithms lead to weighted graphs with the same symmetries; see Figures 2 and 3 .

Example I.3. (Suggested by Mark Jerrum.) Let $K_{n}-K_{n}$ be two copies of the complete graph $K_{n}$ joined by adding an extra edge as in Figures 4 and 5 for $n=4$. Here, the max-degree weights (shown in Figure 5) are dominated by the choice of weights shown in Figure 4. Our numerical results show that the optimal choice differs only slightly from the choice in Figure 4.

In Section 2, we review the classical connections between the spectrum of a self adjoint operator and the representation theory of a group commuting with the operator. Examples 1 and 2 described above are treated. We also review the literature on coherent configurations and the centralizer algebra.

Section 3 gives our first new results. We show how the orbits of various subgroups of the full automorphism group give smaller "orbit chains" that contain 
all the eigenvalues of the original chain. A key result is a useful sufficient condition for an eigenvector of $K$ to descend to an orbit chain. One consequence is a simple way of determining which orbit chains are needed.

In Section 4 the random walk on $F_{m n}$ is explicitly diagonalized. Using all the eigenvalues and eigenvectors, we show that order $n^{2} \log m$ steps are necessary and sufficient to achieve convergence to stationarity in chi-square distance while order $n^{2}$ steps are necessary and sufficient to achieve stationarity in $L^{1}$. In Section 5 all the eigenvalues for any symmetric weights on $K_{n}-K_{n}$ are determined. In Section 6 symmetry analysis is combined with geometric techniques to get good bounds on the weighted chains for $F_{m n}$ (Figures 2 and 3 ). These show that the Metropolis chain is better (by a factor of $\min \{m, n\}$ ) than the max-degree chain. As shown in [Boyd et al. 04], this is the best possible chain.

For background on graph eigenvalues, automorphisms, and their interaction, see [Babai 95], [Chung 97], [Cvetković et al. 95], [Godsil and Royle 01], or [Lauri and Scapellato 03].

\section{Background in Representation Theory}

\section{I Representation Theory}

The interaction of spectral analysis of a self adjoint operator with the representation theory of a group of commuting operators is classical (see [Mackey 78, pages 17-18], Fässler and Stiefel [Fässler and Stiefel 92, pp. 40-43], and Sternberg [Sternberg 94]). Graph theoretic treatment appears in [Cvetković et al. 95, Chapter 5]. The results of this section use the language of elementary representation theory. The references above, or Chapter 1 of [Diaconis 88], give all definitions and many examples.

In present notation, for $K, \pi$ defined in (1.1) and (1.2), let $G$ be a group of automorphisms and $T$ the representation of $G$ on $L^{2}(\pi)$. If $\lambda$ is an eigenvalue of $K$ with eigenspace $M_{\lambda}=\{f \mid K f=\lambda f\}$, then

$$
L^{2}(\pi)=\bigoplus_{\lambda} M_{\lambda}
$$

where the sum is over distinct eigenvalues of $K$. Of course, $L^{2}(\pi)=\bigoplus_{i} V_{i}$ with $V_{i}$ some choice of irreducible representations of $G$. Since $T_{g} M_{\lambda}=M_{\lambda}$, these combine to give

$$
L^{2}=\bigoplus M_{\lambda, i}
$$

with the sum over distinct eigenvalues $\lambda$ and then over irreducible representations of $G, M_{\lambda, i}$ in the eigenspace $M_{\lambda}$. 
Proposition 2.I. (Example I.2, $\boldsymbol{F}_{\boldsymbol{m n n}}$.) For the "flower" $F_{m n}$ defined in Section 1,

(a) the automorphism group is $B_{m}=S_{m} \ltimes C_{2}^{m}$, the hyperoctahedral group;

(b) for $n$ odd,

$$
L^{2}(\pi)=L_{0} \bigoplus_{i=1}^{(n-1) / 2}\left(L_{i 0} \bigoplus L_{i 1} \bigoplus L_{i 2}\right)
$$

with $L_{0}, L_{i 0}$ copies of the one-dimensional trivial representation, $L_{i 1}$ copies of the $(m-1)$-dimensional permutation representation, and $L_{i 2}$ copies of the $m$-dimensional reflection representation of $B_{m}$;

(c) for $n$ even,

$$
L^{2}(\pi)=L_{0} \bigoplus L_{*} \bigoplus_{i=1}^{(n-2) / 2}\left(L_{i 0} \bigoplus L_{i 1} \bigoplus L_{i 2}\right)
$$

with notation as in (b) and $L_{*}=L_{* 0} \bigoplus L_{* 1}$, where $L_{* 0}$ is the one-dimensional trivial representation and $L_{* 1}$ is the $(m-1)$-dimensional permutation representation.

Corollary 2.2. For any choice of invariant weights (with loops allowed), the corresponding Markov chain on $F_{m n}$ has

- for $n$ odd

$$
\begin{array}{rl}
1+(n-1) / 2 & \text { one-dimensional eigenspaces } \\
(n-1) / 2 & (m-1) \text {-dimensional eigenspaces } \\
(n-1) / 2 & m \text {-dimensional eigenspaces }
\end{array}
$$

- for $n$ even

$$
\begin{array}{rl}
1+n / 2 & \text { one-dimensional eigenspaces } \\
n / 2 & (m-1) \text {-dimensional eigenspaces, and } \\
-1+n / 2 & m \text {-dimensional eigenspaces }
\end{array}
$$

Remark 2.3. Of course, in nongeneric situations, some of these eigenspaces may coalesce further. In Section 4, the chain with edge-weights all equal to one is explicitly diagonalized. 
Proof. Label the vertices of $F_{m n}$ as 0 (center) and $(i, j), 1 \leq i \leq m, 1 \leq j \leq n-1$. The hyperoctahedral group $B_{m}=S_{m} \ltimes C_{2}^{m}$ is the group of symmetries of an $m$-dimensional hypercube. Elements are written $(\pi ; x)$ with $\pi \in S_{m}$ permuting the petals ( $i$-variables) and $x=\left(x_{1}, \ldots, x_{m}\right)$ with $x_{i}= \pm 1$, the reflections in the $i$ th petal. Thus, $(\pi ; x)(0)=0$ and $(\pi ; x)(i, j)=\left(\pi(i), x_{i} j\right)$ with operations in the second coordinate carried out modulo $n$. From this, $(\pi ; x)(\sigma ; y)=\left(\pi \sigma ; x^{\sigma} y\right)$ with $x^{\sigma}=\left(x_{\sigma(1)}, x_{\sigma(2)}, \ldots, x_{\sigma(m)}\right)$. This is a standard representation of $B_{m}$. This proves (a). For background on $B_{m}$, see [James and Kerber 81] or [Halverson and Ram 96].

To prove (b), note that the symmetry group splits the vertex set into orbits. These are the central point 0 , the $2 m$ points at distance one away, the $2 m$ points at distance two away, and so on. If $n$ is even, there are only $m$ points at distance $n / 2$. We focus on $n$ odd for the rest of the proof. Thus, $L^{2}(\pi)=L_{0} \bigoplus_{i=1}^{(n-1) / 2} L_{i}$ with $L_{0}$ the one-dimensional trivial representation and $L_{i}$ the $2 m$-dimensional real vector spaces of functions that vanish off the corresponding orbits. All of these $L_{i}, 1 \leq i \leq(n-1) / 2$, are isomorphic representations of $B_{m}$. To decompose into irreducibles, let $e_{1}, e_{2}, \ldots, e_{2 m-1}, e_{2 m}$ be the usual basis for $\mathbf{R}^{2 m}$. The group $B_{m}$ acts on ordered pairs $\left(e_{1}, e_{2}\right),\left(e_{3}, e_{4}\right), \ldots,\left(e_{2 m-1}, e_{2 m}\right)$ by permuting pairs using $\pi$ and using \pm 1 to switch within a pair. Using this, the character $\chi_{2 m}$ on any of these $L_{i}$ is

$$
\chi_{2 m}(\pi ; x)=\sum_{i=1}^{m} \delta_{i \pi(i)}\left(1+x_{i}\right),
$$

where $\delta_{i j}=1$ if $i=j$ and equals zero otherwise. Indeed, $\chi_{2 m}$ is simply the trace of a permutation representation. Now, $\sum \delta_{i \pi(i)}$ is the number of fixed points of $\pi$. This is the usual permutation character of the subgroup $S_{m}$ extended to $B_{m}$. It splits into a one-dimensional trivial representation (character $\chi_{0}$ ) and an $(m-1)$-dimensional irreducible (character $\left.\chi_{m-1}\right)$. Finally, $\sum \delta_{i \pi(i)} x_{i}$ is the character of the usual $m$-dimensional reflection representation of $B_{m}$ acting on $\mathbf{R}^{2 m}$ by permuting coordinates and reflecting in each coordinate. It is easy to show that this is irreducible, e.g., by computing its inner product with itself. Thus, as claimed, $\chi_{2 m}=\chi_{0}+\chi_{m-1}+\chi_{m}$. This holds for each $L_{i}$ proving (b). The proof of (c) is similar.

Proposition 2.4. (Example 1.3, $\boldsymbol{K}_{\boldsymbol{n}}-\boldsymbol{K}_{\boldsymbol{n}}$ ). For two copies of the complete graph $K_{n}$ joined via an extra edge, defined in Section 1,

(a) the symmetry group is $G=C_{2} \ltimes\left(S_{n-1} \times S_{n-1}\right)$.

(b) $L^{2}(\pi)=2 L_{r} \bigoplus L_{2 n-4}$ with $L_{r}$ the two-dimensional regular representation of $C_{2}$ extended to $G$ and $L_{2 n-4}$ an irreducible representation of dimension $2 n-4$. 
Corollary 2.5. For any choice of invariant weights (with loops allowed), the corresponding Markov chain on $K_{n}-K_{n}$ has at most five distinct eigenvalues with one eigenvalue of multiplicity $2 n-4$.

Proof. It is clear by inspection that the automorphisms are all possible permutations of the two sets of $n-1$ vertices, distinct from the connecting edge, among themselves (this gives an action of $S_{n-1} \times S_{n-1}$ ) and switching the two halves (this gives an action of $C_{2}$ ). The actions do not commute and the combined action is the semidirect product $C_{2} \ltimes\left(S_{n-1} \times S_{n-1}\right)$. This proves (a).

To prove (b), observe first that under $G$ there are two orbits: the two points connected by the extra edge and the remaining $2 n-2$ points. The representation of $G$ on the two-point orbit gives one copy of the regular representation of $C_{2}$. Let $\chi$ be the character of the representation of $G$ on the remaining $2 n-2$ points. As a permutation character it is clear that

$$
\chi(x ; \sigma, \zeta)=\delta_{1 x}(\mathrm{FP}(\sigma)+\mathrm{FP}(\zeta))
$$

with $\delta_{1 x}$ being 1 or 0 as $x$ is 1 or -1 and $\operatorname{FP}(\sigma)$ the number of fixed points in $\sigma$. Computing the inner product of $\chi$ with itself gives

$$
\begin{aligned}
\langle\chi, \chi\rangle & =\frac{1}{2((n-1) !)^{2}} \sum_{x, \sigma, \zeta}\left(\delta_{1 x}(\mathrm{FP}(\sigma)+\mathrm{FP}(\zeta))\right)^{2} \\
& =\frac{1}{2((n-1) !)^{2}} \sum_{\sigma, \zeta}\left(\operatorname{FP}^{2}(\sigma)+2 \mathrm{FP}(\sigma) \mathrm{FP}(\zeta)+\mathrm{FP}^{2}(\zeta)\right) \\
& =\frac{1}{2}(2+2+2)=3 .
\end{aligned}
$$

The second from last equality follows by interpreting the sum as an inner product of characters on $S_{n-1} \times S_{n-1}$ and decomposing $\operatorname{FP}(\sigma)$ as a sum of two irreducibles. Thus $\chi$ decomposes as a sum of three irreducibles of $G$. If $\chi_{1}$ and $\chi_{-1}$ are the two characters of $C_{2}$ extended to $G$, computing as above gives $\left\langle\chi, \chi_{1}\right\rangle=\left\langle\chi, \chi_{-1}\right\rangle=1$. It follows that what is left is a $(2 n-4)$-dimensional irreducible of $G$. This proves (b).

Remark 2.6. The irreducible characters of Wreath products such as $G$ are explicitly described in [James and Kerber 81, Chapter 4]. For our special case the irreducible of $G$ having dimension $2 n-4$ may be seen as induced from the $(n-2)$ dimensional representation of $S_{n-1} \times S_{n-1}$. The eigenvalues for all invariant weightings of $K_{n}-K_{n}$ are given in Section 5 . 


\subsection{Centralizer Algebras}

In our work we often begin with a single weighted graph or Markov chain, calculate its symmetry group, and use this to aid in diagonalizing the chain. As the examples of Figures 1-5 show, there are often several chains of interest with the same symmetry group. It is natural to study all weightings consistent with a given symmetry group. This brings us close to the rich world of coherent configurations and distance regular graphs. To see the connection, let $V$ be a finite set and $G$ a group of permutations of $V$. Let $\Omega_{1}, \Omega_{2}, \ldots$ be the orbits of $G$ operating coordinate-wise on $V \times V$. If $A_{i}$ is a $|V| \times|V|$ matrix with $\left(v, v^{\prime}\right)$ entry one or zero as $\left(v, v^{\prime}\right) \in \Omega_{i}$ or not, then the matrices $A_{i}$ satisfy the following:

(1) $\sum A_{i}=J$ (the matrix of all ones),

(2) there is a subset $S$ with $\sum_{i \in S} A_{i}=I$ (the identity),

(3) the set $\left\{A_{i}\right\}$ is closed under taking transposes,

(4) there are numbers $p_{i j}^{k}$ so that $A_{i} A_{j}=\sum_{k} p_{i j}^{k} A_{k}$.

A collection of zero-one matrices satisfying (1)-(4) is called a coherent configuration. Cameron [Cameron 99, Cameron 03] gives a very clear development with extensive references and connections to association schemes, distance regular graphs and much else. Applications to optimization are developed by Gatermann and Parrilo [Gatermann and Parrilo 04]. From (4), the set of real linear combinations of the $A_{i}$ spans an algebra, the centralizer algebra of the action of $G$ on $V$.

The direct connection with our work is as follows: given a graph $(V, E)$ with automorphism group $G$, the set of all labelings of the edges compatible with $G$ gives a subalgebra of the centralizer algebra. The set of all nonnegative weightings gives a convex cone in this subalgebra. The set of all $G$-invariant Markov chains with a fixed stationary distribution is a convex subset of this cone.

We have not found the elegant developments of this theory particularly helpful in our work - we are usually interested in nontransitive actions and use eigenvalues to bound rates of convergence rather than to show that a certain configuration cannot exist. An extremely fruitful application of distance regular graphs to random walk is in [Belsley 98]. It is a natural project to extend Belsley's development to completely general coherent configurations. We may also hope for some synergy between the coding and design developments of Delsrate and the semi-definite tools of [Boyd et al. 04, Gatermann and Parrilo 04]. 
To conclude this section on a more positive note, we give the following proposition:

Proposition 2.7. Let $V$ be a finite set with $G$ a finite group acting on $V$. The set of all Markov chains on $V$ that commute with the action of $G$ is a convex set with extreme points indexed by orbits of $G$ on $(V \times V)$. Given such an orbit, the associated extremal chain is constant in positions $\left(v, v^{\prime}\right)$ in the orbit and has ones on the diagonal of the other rows.

Proof. The only thing to prove is that the construction unambiguously specifies a stochastic matrix. For this, consider rows indexed by $v, v^{\prime}$ that have no diagonal entries. We show that the number of nonzero pairs $(v, w)$ in the orbit is the same as the number of nonzero pairs $\left(v^{\prime}, w^{\prime}\right)$ in the orbit. Suppose that the orbit is $\{g x, g y\}$ for fixed $x \neq y$. Then, $v=g x$ and $v^{\prime}=g^{\prime} x$, so $g^{\prime} g^{-1} v=v^{\prime}$. It follows that if there are $k$ nonzero entries in row $v$, there are $k$ nonzero entries in row $v^{\prime}$.

\section{Orbit Theory}

Let $K, \pi$ be a reversible Markov chain as in (1.1) and (1.2), with $H$ a group of automorphisms. Often, it is a subgroup of the full automorphism group. The vertex set $V$ partitions into orbits $O_{v}=\{h v: h \in H\}$. Define an orbit chain by

$$
K_{H}\left(O_{v}, O_{v^{\prime}}\right)=K\left(v, O_{v^{\prime}}\right)=\sum_{u \in O_{v^{\prime}}} K(v, u)
$$

Note that this is well defined (it is independent of which $v \in O_{v}$ is chosen). Further, the lumped chain (which just reports in which orbit the original chain is) is Markov, with $K_{H}\left(O_{v}, O_{v^{\prime}}\right)$ as the transition kernel. This follows from what is commonly called Dynkin's criteria (the lumped chain is Markov if and only if $K\left(u, O_{v^{\prime}}\right)$ doesn't depend on the choice of $u$ in $\left.O_{v}\right)$. See [Kemeney and Snell 60, Chapter 3] for background. Finally, the chain in (3.1) is reversible with $\pi\left(O_{v}\right)=\sum_{u \in O_{v}} \pi(u)$ as the reversing measure; as a check,

$$
\begin{aligned}
\pi(O) K_{H}\left(O, O^{\prime}\right) & =\sum_{v \in O} \pi(v) K\left(v, O^{\prime}\right)=\sum_{v \in O} \sum_{v^{\prime} \in O^{\prime}} \pi(v) K\left(v, v^{\prime}\right) \\
& =\sum_{v \in O, v^{\prime} \in O^{\prime}} \pi\left(v^{\prime}\right) K\left(v^{\prime}, v\right)=\sum_{v^{\prime} \in O^{\prime}} \pi\left(v^{\prime}\right) K\left(v^{\prime}, O\right) \\
& =\pi\left(O^{\prime}\right) K_{H}\left(O^{\prime}, O\right) .
\end{aligned}
$$


In this section we relate the eigenvalues and eigenvectors of various orbit chains to the eigenvalues and eigenvectors of $K$. This material is related to material surveyed by Chan and Godsil [Chan and Godsil 97], but we have not found our results in other literature.

\section{I Lifting}

Proposition 3.I. Let $K, \pi$ be a reversible Markov chain with automorphism group $G$. Let $H \subseteq G$ be a subgroup. Let $K_{H}$ be defined as in (3.1).

(a) If $\bar{f}$ is an eigenfunction of $K_{H}$ with eigenvalue $\bar{\lambda}$, then $\bar{\lambda}$ is an eigenvalue of $K$ with $H$-invariant eigenfunction $f(v)=\bar{f}\left(O_{v}\right)$.

(b) Conversely, every $H$-invariant eigenfunction appears uniquely from this construction.

Proof. For (a), we just check that, with $f$ as given,

$$
\sum_{v^{\prime}} K\left(v, v^{\prime}\right) f\left(v^{\prime}\right)=\sum_{O^{\prime}} K\left(v, O^{\prime}\right) \bar{f}\left(O^{\prime}\right)=\sum_{O^{\prime}} K\left(O, O^{\prime}\right) \bar{f}\left(O^{\prime}\right)=\bar{\lambda} \bar{f}(O)=\bar{\lambda} f(v) .
$$

For (b), we just check that the only $H$-invariant eigenfunctions occur from this construction. This is precisely the content of "the lemma that is not Burnside's"; see [Neumann 79]. The representation of $H$ on $L^{2}(\pi)$ is the permutation representation corresponding to the action of $H$ on $V$. An $H$-fixed vector $f \in L^{2}(\pi)$ corresponds to a copy of the trivial representation. The character $\chi$ of the representation of $H$ on $L^{2}(\pi)$ is $\chi(h)=\operatorname{FP}(h)=\#\{v \in V: h v=v\}$. "Burnside's lemma" (or Frobenius reciprocity) says that

$$
\frac{1}{|H|} \sum_{h} \mathrm{FP}(h)=\# \text { orbits. }
$$

The left side is the inner product of $\chi$ with the trivial representation. It thus counts the number of $H$-fixed vectors in $L^{2}(\pi)$. The right side counts the number of eigenvalues in the orbit chain. Of course, any $H$-invariant eigenfunction of $K$ projects to a nonzero eigenfunction of the orbit chain (see Proposition 3.3 below).

Remark 3.2. We originally hoped to use the orbit chain under the full automorphism group coupled with the multiplicity information of section two to completely diagonalize the chain. To see how wrong this is, consider a graph such as the complete graph $K_{n}$ with automorphism group operating transitively on $V$. 
Then, the orbit chain has just one point and one $G$-invariant eigenfunction corresponding to eigenvalue one. For the flower $F_{m n}$, with edge weights one, the $C_{2}^{m}$ action collapses each petal into a path (with a loop at the end if $n$ is odd) and then the $S_{m}$ action identifies these paths. It follows that the orbit chain corresponds to unweighted random walk on the path shown in Figure 6.

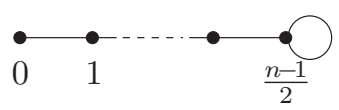

Figure 6. The orbit chain of $F_{m n}$.

It is easy to diagonalize this orbit chain and find the $1+(n-1) / 2$ eigenvalues $\cos (2 \pi j / n), 0 \leq j \leq(n-1) / 2$ (see Section 3.3). These appear with multiplicity one for generic weights. As shown in Section 4 , for weight one, there are non- $G$ invariant eigenvectors with these same eigenvalues, and many further eigenvalues of the full Markov chain $K$.

\subsection{Projection}

As above, $G$ is the automorphism group of a reversible Markov chain, $H \subseteq G$ is a subgroup, and $K_{H}\left(O, O^{\prime}\right)$ is the orbit chain of (3.1). We give a useful condition for an eigenfunction of $K$ to project down to a nonzero eigenfunction of $K_{H}$. Several examples and applications follow.

Proposition 3.3. If $f$ is an eigenfunction of $K$ with eigenvalue $\lambda$, let $\bar{f}(x)=$ $\sum_{h \in H} f\left(h^{-1} x\right)$. If $\bar{f} \neq 0$, then $\bar{f}$ is an eigenfunction for $K_{H}$ with eigenvalue $\lambda$.

Proof. For any $H$-orbit $O$ write $\bar{f}(O)$ for the constant value of $\bar{f}$. For $x \in O$ and $y_{i} \in O_{i}$,

$$
\begin{aligned}
\sum_{i} K\left(O, O_{i}\right) \bar{f}\left(O_{i}\right) & =\sum_{i}\left(\sum_{y \in O_{i}} K(x, y)\right) \sum_{h} f\left(h^{-1} y_{i}\right) \\
& =\sum_{h} \sum_{i} \sum_{y \in O_{i}} K(x, y) f\left(h^{-1} y\right) \\
& =\sum_{h} \sum_{y} K(x, y) f\left(h^{-1} y\right)=\lambda \sum_{h} f\left(h^{-1} x\right)=\lambda \bar{f}(O) .
\end{aligned}
$$


Warning. Of course, $\bar{f}$ can vanish, for example, if the original graph is the cycle $C_{9}$ and $H=C_{3}$ acting by $T_{a}(j)=j+3 a$, for $a \in C_{3}=\{0,1,2\}$. There are 9 original eigenvalues with eigenfunctions $f_{j}(k)=e^{2 \pi i j k / 9}$ (here $i=\sqrt{-1}$ ). Using $\bar{f}_{j}$ as in the proposition above

$$
\begin{aligned}
\bar{f}_{j}(k) & =e^{2 \pi i j k / 9}+e^{2 \pi i j(k+3) / 9}+e^{2 \pi i j(k+6) / 9} \\
& =e^{2 \pi i j k / 9}\left(1+e^{2 \pi i 3 j / 9}+e^{2 \pi i 6 j / 9}\right) \\
& =0 \text { if } j \text { is relatively prime to } 9 .
\end{aligned}
$$

In Case C in Section 3.3 we give examples where several different eigenfunctions coalesce under projection. The following proposition gives sufficient conditions for an eigenvalue of $K$ to appear in a projection.

Proposition 3.4. Let $H$ be a subgroup of the automorphism group $G$ of a reversible Markov chain $K$. Let $f$ be an eigenfunction of $K$ with eigenvalue $\lambda$. Then $\lambda$ appears as an eigenvalue in $K_{H}$ if either of the following conditions holds:

(a) $H$ has a fixed point $v^{*}$ and $f\left(v^{*}\right) \neq 0$, or

(b) $f$ is nonzero at $v^{*}$ that is in a G-orbit containing an $H$ fixed point.

Proof. For (a), $\bar{f}$ defined in Proposition 3.3 satisfies $\bar{f}\left(O_{v^{*}}\right) \neq 0$ because $\bar{f}\left(O_{v^{*}}\right)=$ $|H| f\left(v^{*}\right)$. For (b), since $f\left(v^{*}\right) \neq 0$, let $g$ map $v^{*}$ to $v^{* *}$ an $H$-fixed point. Then, $\overline{\left(T_{g} f\right)}\left(v^{*}\right) \neq 0$.

Example 3.5. $\left(\boldsymbol{F}_{\boldsymbol{m} \boldsymbol{n}}\right.$. $)$ Let $H=B_{m-1}$, the subgroup of $B_{m}$ fixing the first petal. The orbit graph is a weighted lollipop $L_{n}$ (see Figure 7). The weights are determined from (3.1), which specifies $\pi(O)$ and $K\left(O, O^{\prime}\right)$. The weight on edge $\left(O, O^{\prime}\right)$ is then $\pi(O) K\left(O, O^{\prime}\right)$ (see (1.2) and (1.3)). We claim that all the eigenvalues of the weight one random walk on $F_{m n}$ occur as eigenvalues of $L_{n}$. Indeed, if $f$ is an eigenfunction of $F_{m n}$ with $f(0) \neq 0$, then we are done by (a) of Proposition 3.4. If $f(0)=0$, then $f(v) \neq 0$ for some other $v$. We may map $v$ to the first petal (fixed by $H$ ). We are done by (b) of Proposition 3.4.
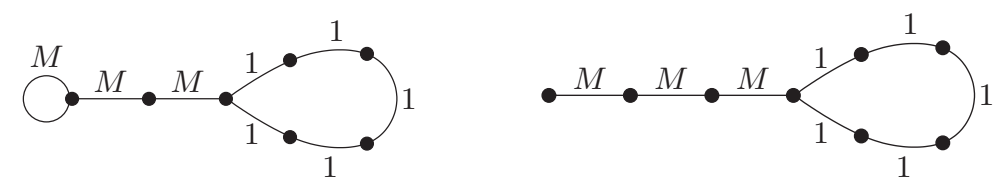

Figure 7. The weighted lollipop graph $L_{n}: n$ odd (left) and $n$ even (right); $M=2(m-1)$. 


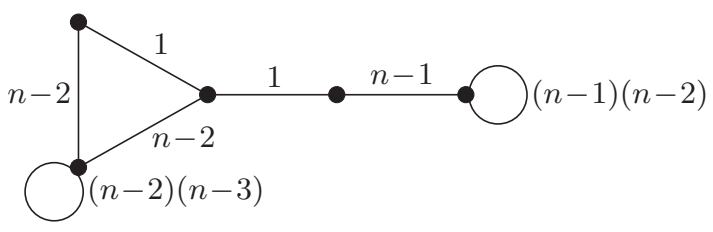

Figure 8. The weighted orbit chain of $K_{n}-K_{n}$ under the group action $S_{n-2} \times S_{n-1}$.

Example 3.6. $\left(\boldsymbol{K}_{\boldsymbol{n}}-\boldsymbol{K}_{\boldsymbol{n}}\right.$.) Consider the subgroup $S_{n-2} \times S_{n-1} \subseteq C_{2} \ltimes\left(S_{n-1} \times S_{n-1}\right)$. The orbit graph is shown in Figure 8. Arguing as above, we see all eigenvalues of the unweighted walk appear.

It is natural to ask which orbit chains are needed to get all the eigenvalues of the original chain $K$. The following theorem gives a simple answer.

Theorem 3.7. Let $G$ be the automorphism group of the reversible Markov chain $(K, \pi)$. Suppose that $V=O_{1} \cup \ldots \cup O_{k}$ as a disjoint union of $G$-orbits. Represent $O_{i}=G / H_{i}$ with $H_{i}$ the subgroup fixing a point in $O_{i}$. Then, all eigenvalues of $K$ occur among the eigenvalues of $\left\{K_{H_{i}}\right\}_{i=1}^{k}$. Further, every eigenfunction of $K$ occurs by translating a lift of an eigenfunction of some $K_{H_{i}}$.

Proof. Say $f$ is an eigenfunction of $K$ with eigenvalue $\lambda$. Let $f(v) \neq 0$, and say $v \in O_{i} \cong G / H_{i}$ with $H_{i}=\left\{h \mid h v_{i}=v_{i}\right\}$ for a prechosen $v_{i} \in O_{i}$. Choose $g$ with $g^{-1} v=v_{i}$, and let $f_{1}=T_{g} f$. Then, $f_{1}$ has $\lambda$ as an eigenvalue and a nonzero $H_{i}$-invariant point. The result follows from proposition (3.4).

Remark 3.8. Observe that if $H \subseteq J \subseteq G$, then the eigenvalues of $K_{H}$ contain all eigenvalues of $K_{J}$. This allows disregarding some of the $H_{i}$. Consider Example $3.5\left(F_{m n}\right)$ with $n$ odd. There are $1+(n-1) / 2$ orbits $O_{0} \cup O_{1} \cup \ldots \cup O_{(n-1) / 2}$. The corresponding $H_{i}$ are $G$ for $O_{0}$ and $B_{m-1}$ for all the other $O_{i}$. It follows that all the eigenvalues occur in the orbit chain for $B_{m-1}$, this is the lollipop $L_{n}$ described above. Similarly, for $K_{n}-K_{n}$, there are two orbits: the two central points (with $H_{1}=S_{n-1} \times S_{n-1}$ ) and the remaining $2 n-2$ points (with $\left.H_{2}=S_{n-2} \times S_{n-1}\right)$. Since $H_{2} \subseteq H_{1}$, we get all eigenvalues from this quotient, as discussed in Theorem 3.7 .

There remains the question of relating the orbit theory of this section with the multiplicity theory coming from the representation theory of Section 2. We have not sorted this out neatly. The following classical proposition gives a simple answer in the transitive case. 
Proposition 3.9. Let $G$ be the automorphism group of the reversible Markov chain $(K, \pi)$. Suppose that $G$ acts transitively on $V$. Let $L^{2}(\pi)=V_{1} \oplus \ldots \bigoplus V_{k}$ be the isotypic decomposition with $V_{i}=d_{i} W_{i}$ and $W_{i}$ distinct irreducible representations. Suppose that $V \cong G / H$. Then, the $H$-orbit chain has $\sum_{i=1}^{k} d_{i}$ distinct eigenvalues generically, with $d_{i}$ eigenvalues having multiplicity $\operatorname{Dim}\left(W_{i}\right)$ in the original chain $K$. These eigenvalues may be determined as follows: set $Q(y)=K(H, y H) /|H|$. This is an $H$-bi-invariant probability measure on $G$ $\left(Q\left(h_{1} g h_{2}\right)=Q(g)\right)$. Let $\rho_{i}$ be a matrix representation for $W_{i}$ with basis chosen so that the first $d_{i}$ basis vectors are fixed by $H$. Then, $\widetilde{Q}\left(\rho_{i}\right)=\sum_{g} Q(g) \rho_{i}(g)$ is zero except for the upper left $d_{i} \times d_{i}$ block. The eigenvalues of this block are the $d_{i}$ eigenvalues, each with multiplicity $\operatorname{Dim}\left(W_{i}\right)$.

Proof. This is standard in the multiplicity free case [Diaconis 88, Chapter 3]. Dieudonne [Dieudonne 78, Section 22.5] covers the general case.

\section{Remark 3.10.}

1. In the transitive case, $L(V)=\operatorname{Ind}_{H}^{G}(1)$. The $H$-orbit chain is indexed by $H$ $H$ double cosets. By the Mackey intertwining theorem [Curtis and Reiner $62,44.5]$, the number of orbits is $\sum d_{i}^{2}$. Thus, the $H$-orbit chain, which has only $\sum d_{i}$ distinct eigenvalues, has the $d_{i}$ eigenvalues each occuring with multiplicity $d_{i}$.

For example, consider the hypercube $C_{2}^{n}$. The automorphism group is $G=B_{n}$, the hyperoctahedral group. This operates transitively with $C_{2}^{n}=$ $G / H$ for $H=S_{n}$. Further, $L^{2}(\pi)=\bigoplus_{i=1}^{n} W_{i}$ with $\operatorname{Dim}\left(W_{i}\right)=\left(\begin{array}{c}n \\ i\end{array}\right)$. As is well known, random walk on $C_{2}^{n}$ has eigenvalues $1-\frac{2 i}{n}, 0 \leq i \leq n$ with multiplicity $\left(\begin{array}{c}n \\ i\end{array}\right)$. See [Diaconis 88 , page 28] for background.

2. In the nontransitive case, Arun Ram has taken us a step closer to connecting the orbit theory to the representation theory. Suppose that, as a representation of $G, L^{2}=\sum_{\lambda} d_{\lambda} V^{\lambda}$, with $V^{\lambda}$ irreducible representations of $G$ occurring with multiplicity $d_{\lambda}$. Let $\mathcal{H}=\operatorname{End}_{G}\left(L^{2}\right)$ be the algebra of all linear transformations that commute with $G$. Then, $L^{2}$ is a $(G, \mathcal{H})$ bi-module, and basic facts about double commutators ([Mackey 78, pages 17-18]) give

$$
L^{2}=\bigoplus_{\lambda} V^{\lambda} \bigotimes W^{\lambda}, \quad \text { as a }(G, \mathcal{H}) \text { bi-module. }
$$

In this decomposition $G$ only acts on $V^{\lambda}$. The $d_{\lambda}$-dimensional space $W^{\lambda}$ is called a multiplicity space. Dually, $\mathcal{H}$ (and so $K$ ) only acts on $W^{\lambda}$, and 
each eigenvalue of $K$ on $W^{\lambda}$ occurs with multiplicity $\operatorname{Dim}\left(V^{\lambda}\right)$. Usually, the action of $K$ on $W^{\lambda}$ (or even an explicit description of $W^{\lambda}$ ) is not apparent.

If $\mathcal{X}=G / H_{1} \cup G / H_{2} \cup \ldots \cup G / H_{r}$ is a union of $G$ orbits, Theorem 3.7 says that we need only consider the $H_{i}$ orbit chains. By standard theory, the $H_{i}$ lumped chain $K_{H_{i}}$ may be seen as the action of $K$ on

$$
L\left(H_{i} \backslash \mathcal{X}\right) \cong \bigoplus_{\lambda}\left(V^{\lambda}\right)^{H_{i}} \bigotimes W^{\lambda}
$$

Here, $\left(V^{\lambda}\right)^{H_{i}}$ is the subspace of $H_{i}$-invariant vectors in the representation of $G$ on $V^{\lambda}$.

The point is that (as in the examples), we may be able to calculate all the eigenvalues of $K_{H_{i}}$. Further, we know that these occur with multiplicity $\operatorname{Dim}\left(\left(V^{\lambda}\right)^{H_{i}}\right)$. These numbers are computable from group theory, independently of $K$. If they are distinct, they allow us to identify the eigenvalues of $K$ on $W^{\lambda}$. For $\lambda$ allowing $H_{i}$-fixed vectors, the action of $K$ on $W^{\lambda}$ is the same in (3.2) and (3.3). With several $H_{i}$, the possibility of unique identification is increased.

3. An example of Ron Graham shows that we should not hope for too much from symmetry analysis. To see this, consider the simplest graph with no symmetry (Figure 9, left). Take $n$ copies of this six vertex graph and join them, head to tail, in a cycle. This $6 n$ vertex graph has only $C_{n}$ symmetry. The orbit graph is shown on the right in Figure 9. By Proposition 3.1, each of the six eigenvalues of this orbit graph occur with multiplicity one in the large graph. We have not found any way to get a neat description of the remaining eigenvalues. The quotient of the characteristic polynomial of the big graph by that of the orbit graph is often irreducible for small examples. Of course, we can get good bounds on the eigenvalues with geometric arguments as in Section 6. However, symmetry does not give complete answers.
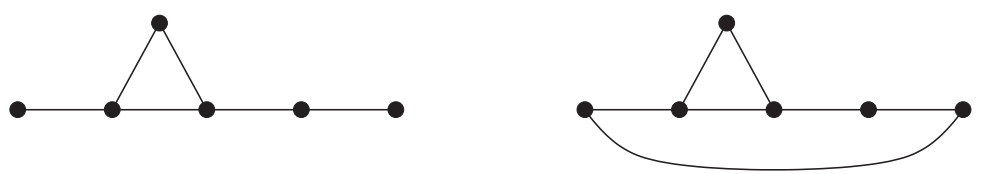

Figure 9. Left: the simplest graph with no symmetry. Right: orbit graph with $C_{n}$ symmetry. 

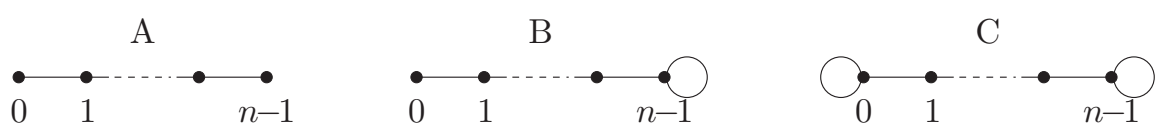

Figure 10. Three path graphs with $n$ vertices.

\subsection{Three $C_{2}$ actions}

We now illustrate the orbit theory for three classical $C_{2}$ actions. The results below are well known, see [Kac 47] or [Feller 68]. We find them instructive in the present context. Further, we need the very detailed description that we provide to diagonalize $F_{m n}$. Pinsky [Pinsky 80, Pinsky 85] gives a much more elaborate example of this type of argument.

Consider the three graphs in Figure 10, each on $n$-vertices. It is well known that the nearest neighbor Markov chain on each can be explicitly diagonalized by lifting to an appropriate circle.

Case A. Consider $C_{2(n-1)}$. For example, Figure 11 shows the case with $n=4$. Label the points of $C_{2(n-1)}$ as $0,1, \ldots, 2(n-1)-1$. Let $C_{2}$ act on $C_{2(n-1)}$ by $j \rightarrow-j$. This fixes 0 and $n-1$ and gives $n-2$ two-point orbits. The orbit chain is precisely the loopless path of Case A in Figure 10. The eigenvalues/functions of $C_{2(n-1)}$ are

$$
\begin{aligned}
1 & / \text { constant } \\
-1 & / \cos \left(\frac{2 \pi(n-1) k}{2(n-1)}\right)=\cos (\pi k) \\
\cos \left(\frac{2 \pi j}{2(n-1)}\right) & / \cos \left(\frac{2 \pi j k}{2(n-1)}\right), \sin \left(\frac{2 \pi j k}{2(n-1)}\right), \quad 1 \leq j \leq n-2 .
\end{aligned}
$$

Using Proposition 3.3, relabeling vertices of the path as $0,1, \ldots, n-1$, we have the following:

Proposition 3.II. The loopless path of length $n$ has eigenvalues $\cos \left(\frac{\pi j}{n-1}\right)$ with eigenfunction $f_{j}(k)=\cos \left(\frac{\pi j k}{n-1}\right), 0 \leq j \leq n-1$.
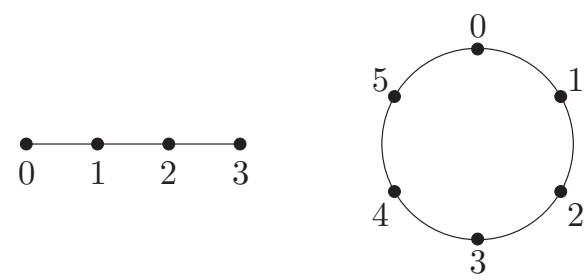

Figure II. Case A, $n=4$. 

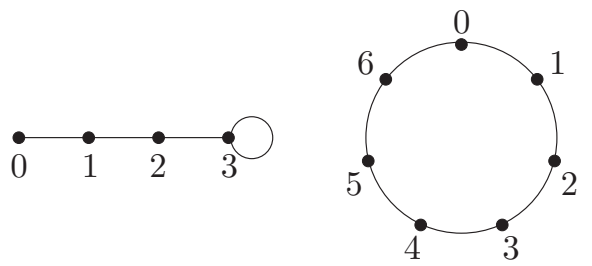

Figure 12. Case $\mathrm{B}, n=4$.

Note. Here -1 is an eigenvalue of the loopless path; all eigenvalues are distinct, and $\cos \left(\frac{\pi j}{n-1}\right)=-\cos \left(\frac{\pi(n-1-j)}{n-1}\right)$.

Case B. Consider $C_{2 n-1}$. For example, Figure 12 shows the case with $n=4$. Again $C_{2}$ acts on $C_{2 n-1}$ by $j \rightarrow-j$. This fixes 0 and there are $n-1$ orbits of size two. The orbit chain is the single loop chain of Case B in Figure 10. The eigenvalue/function pairs of $C_{2 n-1}$ are

$$
\begin{aligned}
1 & / \text { constant } \\
\cos \left(\frac{2 \pi j}{2 n-1}\right) & / \cos \left(\frac{2 \pi j k}{2 n-1}\right), \quad \sin \left(\frac{2 \pi j k}{2 n-1}\right), \quad 1 \leq j \leq n-1 .
\end{aligned}
$$

Proposition 3.I2. The single loop path of Case B has eigenvalues $\cos \left(\frac{2 \pi j}{2 n-1}\right)$ with eigenfunction $f_{j}(k)=\cos \left(\frac{2 \pi j k}{2 n-1}\right), 0 \leq j \leq n-1$.

Note. Here -1 is not an eigenvalue, and all eigenvalues have multiplicity one.

Case C. Consider $C_{2 n}$. For example, Figure 13 show the case with $n=4$. Map $C_{2 n} \rightarrow C_{2 n}$ with $T(k)=2 n-1-k=-(k+1), 0 \leq k \leq 2 n-1$. Clearly, $T^{2}(k)=k$ and $T$ sends edges to edges. There are $n$ orbits of size two. The orbit chain is the double loop chain of Case $\mathrm{C}$ in Figure 10. The eigenvalue/function
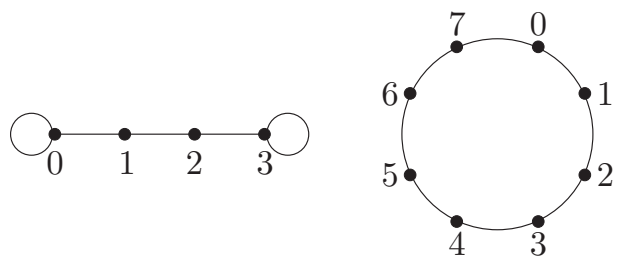

Figure 13. Case $\mathrm{C}, n=4$. 
pairs of $C_{2 n}$ are

$$
\begin{array}{rll}
1 & / & \text { constant } \\
-1 & / \cos (\pi k) \\
\cos \left(\frac{2 \pi j}{2 n}\right) & / \cos \left(\frac{2 \pi j k}{2 n}\right), \quad \sin \left(\frac{2 \pi j k}{2 n}\right), \quad 1 \leq j \leq n-1 .
\end{array}
$$

Summing over orbits gives the next proposition.

Proposition 3.13. The double loop path of length $n$ has eigenvalues $\cos \left(\frac{\pi j}{n}\right)$ with eigenfunction

$$
f_{j}(k)=\cos \left(\frac{\pi j k}{n}\right)+\cos \left(\frac{\pi j(k+1)}{n}\right),
$$

$0 \leq j \leq n-1,0 \leq k \leq n-1$. Using $\cos (x)+\cos (y)=2 \cos \left(\frac{x+y}{2}\right) \cos \left(\frac{x-y}{2}\right)$, we see that $f_{j}(k)$ is proportional to $\cos \left(\frac{\pi j}{n}\left(k+\frac{1}{2}\right)\right)$.

Remark 3.I4. In this example, we may also write

$$
f_{j}(k)=\sin \left(\frac{\pi j k}{n}\right)-\sin \left(\frac{\pi j(k+1)}{n}\right),
$$

$0 \leq j \leq n-1$. Note that

$$
\sin \left(\frac{\pi j k}{n}\right)-\sin \left(\frac{\pi j(k+1)}{n}\right)=\left(\cos \left(\frac{\pi j k}{n}\right)+\cos \left(\frac{\pi j(k+1)}{n}\right)\right)\left(-\tan \left(\frac{\pi j}{2 n}\right)\right) .
$$

This shows another way that eigenfunctions can collapse. On $C_{2 n}$ we may choose the pairing $T(k)=a-k$, for any odd $a$ (even $a$ leads to fixed points).

\section{Simple Random Walk on $\boldsymbol{F}_{\boldsymbol{m} n}$}

In this section we give an explicit diagonalization of the random walk on the "flower" $F_{m n}$ with all edge weights one. We have two motivations: first, to give an illustration of the theory developed in a two parameter family of examples and second, the analysis of rates of convergence of the random walk to stationarity needs both eigenvalues and eigenvectors. It clears up a mystery that was troubling us in comparing different weighted walks on $F_{m n}$. Our careful analysis allows us to show that the walks have different rates of convergence in $L^{2}$ and $L^{1}-n^{2} \log m$ vs $n^{2}$. We first give the diagonalization, then the $L^{2}$ analysis, then the $L^{1}$ analysis. We note that the graph $F_{m 3}$ is thoroughly studied as the "friendship graph"; see [Erdös et al. 66]. 


\section{I Diagonalizing Simple Random Walk on $\boldsymbol{F}_{\boldsymbol{m} n}$}

The walk $K\left(v, v^{\prime}\right)$ and stationary distribution $\pi(v)$ were introduced in Section 1. We suppose throughout this section that $n \geq 3$ is odd and $m \geq 2$ is arbitrary. The state-space has $|V|=1+m(n-1)$.

Proposition 4.I. For $n \geq 3$ odd and $m \geq 2$, let $K\left(v, v^{\prime}\right)$ be a simple random walk on the flower $F_{m n}$ with points $0,(i, j), 1 \leq i \leq m, 1 \leq j \leq n-1$. The walk is reversible with stationary distribution $\pi(0)=\frac{1}{n}, \pi(i, j)=\frac{1}{m n}$. The eigenvalues $\lambda$ and an orthonormal basis of eigenvectors $f(i, j)$ are

$$
\begin{aligned}
& 1 \text { / constant } \\
& \cos \left(\frac{2 \pi a}{n}\right) \quad / \quad \sqrt{2} \cos \left(\frac{2 \pi a j}{n}\right), \quad 1 \leq a \leq \frac{n-1}{2} \\
& \cos \left(\frac{2 \pi a}{n}\right) \quad / \quad \sqrt{2 m} s_{a b}(i, j), \quad 1 \leq a \leq \frac{n-1}{2}, \quad 1 \leq b \leq m \\
& \cos \left(\frac{\pi(2 a+1)}{n}\right) \quad / \quad \sqrt{2 m} \sqrt{\frac{b}{b+1}} f_{a b}(i, j), \quad 0 \leq a \leq \frac{n-3}{2}, \quad 1 \leq b \leq m-1,
\end{aligned}
$$

where

$$
\begin{aligned}
& s_{a b}(i, j)= \begin{cases}\sin \left(\frac{2 \pi a j}{n}\right) & \text { if } i=b \\
0 & \text { if } i \neq b\end{cases} \\
& f_{a b}(i, j)= \begin{cases}\frac{1}{b} \cos \left(\frac{\pi(2 a+1)}{n}\left(\frac{n}{2}-j\right)\right) & \text { if } 1 \leq i \leq b \\
\cos \left(\frac{\pi(2 a+1)}{n}\left(\frac{n}{2}+j\right)\right) & \text { if } i=b+1 \\
0 & \text { if } b+1<i \leq m .\end{cases}
\end{aligned}
$$

Remark 4.2. This gives $1+(n-1) / 2+m(n-1) / 2+(m-1)(n-1) / 2=1+m(n-1)$ pairs $\lambda / f$. Comparing with Corollary 2.2, we see that the multiplicities check but the eigenvalues for the one-dimensional spaces sometimes equal the eigenvalues for the $(m-1)$-dimensional eigenspaces. In Section 6 , with weights on the edges to force a uniform distribution, all these "accidents" disappear. To evaluate the eigenfunctions at zero, use the expressions given with $j=0$.

Proof. We lift eigenvalues from two distinct orbit chains: a cycle $C_{n}$ (with $H=$ $S_{m}$ ) and a path with loops (with $H=S_{m-1} \ltimes C_{2}^{m}$ ). The argument breaks into the following cases:

- vectors coming from the circle $C_{n}$ not vanishing at zero,

- vectors coming from the circle $C_{n}$ vanishing at zero and their shifts,

- vectors coming from a path and their shifts. 
The results are developed in this order.

(a) Vectors coming from $C_{n}$. The symmetric group $S_{m}$ acts on $F_{m n}$ and the orbit chain is the simple random walk on $C_{n}$. This has eigenvalues/vectors

$$
\begin{aligned}
1 & / \text { constant } \\
\cos \left(\frac{2 \pi a}{n}\right) & / \quad f_{a}(j)=\cos \left(\frac{2 \pi a j}{n}\right), \quad \sin \left(\frac{2 \pi a j}{n}\right), \quad 1 \leq a \leq \frac{n-1}{2} .
\end{aligned}
$$

We lift these eigenvectors up to $F_{m, n}$ in two ways:

(1) The eigenvectors $\cos (2 \pi a j / n)$ are lifted to $F_{m n}$ by defining them to be constant on orbits of $S_{m}$. This gives $(n-1) / 2$ eigenvectors, each associated with a distinct eigenvalue. Note that since $\cos (2 \pi a j / n)=$ $\cos (-2 \pi a j / n)$ for all $j$, these are in fact $B_{m}$ invariant and exactly the eigenvectors accounted for by Proposition 3.1. By an elementary computation

$$
\lambda_{a}=\cos \left(\frac{2 \pi a}{n}\right), \quad f_{a}(i, j)=\sqrt{2} \cos \left(\frac{2 \pi a j}{n}\right)
$$

are orthonormal eigen pairs.

(2) The eigenvectors $\sin (2 \pi a j / n)$ vanish at $j=0$. Because of this, we may define $m$ distinct lifts by installing $\sin (2 \pi a j / n)$ on the $b$ th petal $(1 \leq b \leq m)$ and defining it as zero elsewhere. Thus, define

$$
s_{a b}(i, j)= \begin{cases}\sin \left(\frac{2 \pi a j}{n}\right) & \text { if } i=b, \\ 0 & \text { if } i \neq b .\end{cases}
$$

It is easy to check that this works: for $i \neq b, j \neq 0$, with $K$ the transition kernel of $F_{m n}$,

$$
K s_{a b}(i, j)=0=\cos \left(\frac{2 \pi a j}{n}\right) s_{a b}(i, j) .
$$

For $i=b, j \neq 0$,

$$
K s_{a b}(i, j)=\cos \left(\frac{2 \pi a j}{n}\right) s_{a b}(i, j) .
$$

Finally at 0 ,

$$
\begin{aligned}
K s_{a b}(0) & =\frac{1}{2 m} \sum_{i}\left(s_{a b}(i, 1)+s_{a b}(i,-1)\right) \\
& =\frac{1}{2 m}\left(s_{a b}(b, 1)+s_{a b}(b,-1)\right)=0=\cos \left(\frac{2 \pi a j}{n}\right) s_{a b}(0) .
\end{aligned}
$$

This gives $m(n-1) / 2$ further eigenvectors that have been normalized in the statement. 


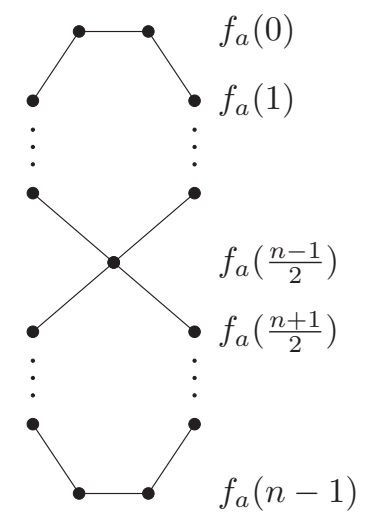

Figure 14. Definition of $f_{a 1}$ on $F_{2 n}$.

(b) Vectors coming from a path. In Section 3.3, Case C, we diagonalized a simple random walk on a path of length $n$ with two loops (Figure 13). We now lift the eigenvalues

$$
\cos \left(\frac{\pi(2 a+1)}{n}\right)
$$

$0 \leq a \leq \frac{n-3}{2}$, and eigenfunctions

$$
f_{a}(k)=\cos \left(\frac{\pi(2 a+1)}{n}\left(k+\frac{1}{2}\right)\right),
$$

$0 \leq k \leq n-1$, up to $F_{m n}$.

We first show the case $m=2$. In this case, we lift $f_{a}$ from a path to $f_{a b}$ $(b=1)$ on two petals as shown in Figure 14, with $f_{a}$ assigned symmetrically on the two halves of the petal. Here, $f_{a}$ is indexed by $k=0,1, \ldots, n-1$ from top to bottom. In terms of $f_{a 1}$ indexed by $(i, j)$ on the petals, this is equivalent to

$$
f_{a 1}(i, j)=\left\{\begin{aligned}
f_{a}\left(\frac{n-1}{2}-j\right) & =\cos \left(\frac{\pi(2 a+1)}{n}\left(\frac{n}{2}-j\right)\right) \\
& \text { if } i=1 \text { (the upper petal) } \\
f_{a}\left(\frac{n-1}{2}+j\right) & =\cos \left(\frac{\pi(2 a+1)}{n}\left(\frac{n}{2}+j\right)\right) \\
& \text { if } i=2 \text { (the lower petal). }
\end{aligned}\right.
$$

It is easy to check that $f_{a b}(i, j)=f_{a 1}(i, n-j)$, which means that $f_{a b}$ is symmetric on the two halves of each petal, as desired. We also notice that $f_{a 1}(1, j)=-f_{a 1}(2, j)$, so the eigenvector is "skew-symmetric" on the two petals. 


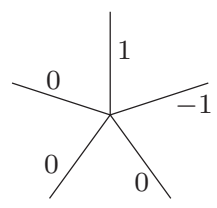

$b=1$

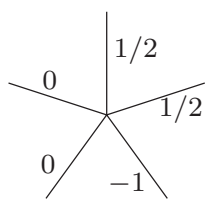

$b=2$

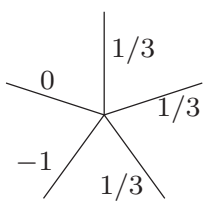

$b=3$

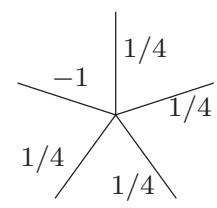

$b=4$

Figure 15. Construction of orthogonal eigenvectors on a spider graph with $m=5$ legs.

For $m>2$, we lift $f_{a}$ up to the graph $F_{m n}$ in $m-1$ ways, indexed by $b=1, \ldots, m-1$. For each $b$, we assign $1 / b$ of the first half of $f_{a}$, $\left\{f_{a}(0), \ldots, f_{a}\left(\frac{n-1}{2}\right)\right\}$, on petals $i=1, \ldots, b$, and assign the second half $\left\{f_{a}\left(\frac{n-1}{2}+1\right), \ldots, f_{a}(n-1)\right\}$ on petal $i=b+1$. The rest of the petals are assigned zero. In other words, we have

$$
f_{a b}(i, j)= \begin{cases}\frac{1}{b} f_{a}\left(\frac{n-1}{2}-j\right)=\frac{1}{b} \cos \left(\frac{\pi(2 a+1)}{n}\left(\frac{n}{2}-j\right)\right) & \text { if } 1 \leq i \leq b \\ f_{a}\left(\frac{n-1}{2}+j\right)=\cos \left(\frac{\pi(2 a+1)}{n}\left(\frac{n}{2}+j\right)\right) & \text { if } i=b+1 \\ 0 & \text { if } b+1<i \leq m .\end{cases}
$$

As before, $f_{a b}$ is symmetric on the two halves of each petal. Because of this symmetry, we need only consider $F_{m n}$ with each petal flattened into a path of length $\frac{n+1}{2}$, all $m$ such paths being joined at an end point. The sign pattern (considering the first half of $f_{a}$ as a unit) when $m=5$ is illustrated in Figure 15. We will refer to this graph as a spider with $m$ legs.

It is easy to see that $f_{a b}, 1 \leq b \leq m-1$, are eigenfunctions with eigenvalue

$$
\cos \left(\frac{\pi(2 a+1)}{n}\right)
$$

see Remark 4.3. We claim that they are mutually orthogonal. Prove this inductively in $b$. If $f_{a 1}, \ldots, f_{a(b-1)}$ are mutually orthogonal, the inner product of $f_{a b}$ with any of these has its negative part dotted with zero. Of the remaining parts, all of the legs of $f_{a b}$ have positive sign. For any $f_{a j}, j \leq b-1$, one of the legs is negative and the others are positive divided by $j$. The sum of the inner products is zero. For $F_{m n}$, the nonuniform stationary probability doesn't affect things because $f_{a b}(i, 0)=0$.

Remark 4.3. Implicit in the above manipulations is the following diagonalization of a random walk on a weighted path with end loops. Consider a path with $n$ vertices, with $n$ odd, and weights $a, b>0$ on the edges as shown in Figure 16 . 


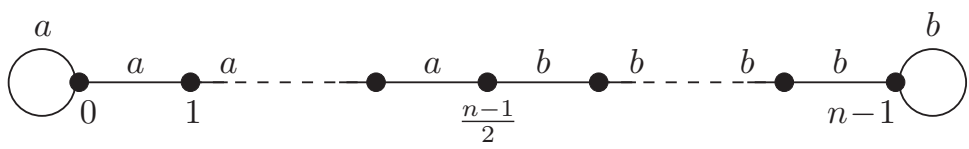

Figure 16. A path with different weights on the left and right.

The nearest neighbor random walk on this graph has stationary distribution

$$
\pi\left(\frac{n-1}{2}\right)=\frac{a+b}{n(a+b)}=\frac{1}{n}, \quad \pi(i)= \begin{cases}\frac{2 a}{n(a+b)}, & 0 \leq i<\frac{n-1}{2} \\ \frac{2 b}{n(a+b)}, & \frac{n-1}{2}<i \leq n-1 .\end{cases}
$$

If $a=b$, the walk is symmetric; the eigenvalues and eigenvectors were determined in Case C of Section 3.3:

$$
\lambda_{j}=\cos \left(\frac{j \pi}{n}\right), \quad f_{j}(k)=\cos \left(\frac{j \pi}{n}\left(k+\frac{1}{2}\right)\right), \quad 0 \leq j, k \leq n-1 .
$$

Proposition 4.4. For any odd $n \geq 3$ and $a, b>0$, the Markov chain $\hat{K}$ on the weighted path of Figure 16 has eigenvalues $\hat{\lambda}_{j}=\lambda_{j}=\cos \left(\frac{j \pi}{n}\right), 0 \leq j \leq n-1$. The eigenvectors are

$$
\begin{array}{ll}
j \text { even } & \hat{f}_{j}(k)=f_{j}(k), \quad 0 \leq k \leq n, \text { see }(4.1) \\
j \text { odd } & \hat{f}_{j}(k)= \begin{cases}f_{j}(k), & 0 \leq k<\frac{n-1}{2} \\
0, & k=\frac{n-1}{2} \\
\frac{a}{b} f_{j}(k), & \frac{n-1}{2}<k \leq n-1 .\end{cases}
\end{array}
$$

Proof. Because the $f_{j}$ are eigenvectors for the stated eigenvalues when $a=b$, we need only check them at $k=\frac{n-1}{2}$. For $j$ even,

$$
\begin{aligned}
\hat{K} \hat{f}\left(\frac{n-1}{2}\right) & =\frac{a}{a+b} \hat{f}_{j}\left(\frac{n-1}{2}-1\right)+\frac{b}{a+b} \hat{f}_{j}\left(\frac{n-1}{2}+1\right) \\
& =\frac{a}{a+b} \cos \left(\frac{j \pi(n-2)}{2 n}\right)+\frac{b}{a+b} \cos \left(\frac{j \pi(n+2)}{2 n}\right) \\
& =\left(\frac{a}{a+b}+\frac{b}{a+b}\right) \cos \left(\frac{j \pi}{n}\right) \cos \left(\frac{j \pi}{2}\right)=\hat{\lambda}_{j} \hat{f}_{j}\left(\frac{n-1}{2}\right) .
\end{aligned}
$$

The next to last equality used $\cos (x+y)=\cos (x) \cos (y)-\sin (x) \sin (y)$. For $j$ odd,

$$
\begin{aligned}
\hat{K} \hat{f}\left(\frac{n-1}{2}\right) & =\frac{a}{a+b} f_{j}\left(\frac{n-1}{2}-1\right)+\frac{b}{a+b} \frac{a}{b} f_{j}\left(\frac{n-1}{2}+1\right) \\
& =\frac{a}{a+b}\left(f_{j}\left(\frac{n-1}{2}-1\right)+f_{j}\left(\frac{n-1}{2}+1\right)\right) \\
& =0=\hat{\lambda}_{j} \hat{f}_{j}\left(\frac{n-1}{2}\right) .
\end{aligned}
$$


Remark 4.5. If $n$ (the number of vertices in one petal) is even, a similar result holds for the path of length $n+1$ without loops (the orbit chain under $H=S_{m-1} \ltimes C_{2}^{m}$ ). From Proposition 3.11, when $a=b$, this graph has eigenvalues $\lambda_{j}=\cos \left(\frac{j \pi}{n}\right)$ and eigenvectors $f_{j}(k)=\cos \left(\frac{j k \pi}{n}\right), 0 \leq j, k \leq n$. For general $a, b>0$, the eigenvalues are $\hat{\lambda}_{j}=\lambda_{j}, 0 \leq j \leq n$ and

$$
\begin{array}{ll}
j \text { even } & \hat{f}_{j}(k)=f_{j}(k), \quad 0 \leq k \leq n \\
j \text { odd } & \hat{f}_{j}(k)= \begin{cases}f_{j}(k), & 0 \leq k<\frac{n}{2} \\
0, & k=\frac{n}{2} \\
\frac{a}{b} f_{j}(k), & \frac{n}{2}<k \leq n .\end{cases}
\end{array}
$$

\subsection{Rates of Convergence}

Theorem 4.6. There exist positive constants $A, B, C$ such that if $K\left(v, v^{\prime}\right)$ denotes the transition matrix of a simple random walk on the unweighted graph $F_{m n}$, then with $m \geq 2, n \geq 3$ odd, for all $v$ and $l \geq 1$,

$$
A e^{-B l /\left(n^{2} \log m\right)} \leq \sum_{v^{\prime}} \frac{\left(K^{l}\left(v, v^{\prime}\right)-\pi\left(v^{\prime}\right)\right)^{2}}{\pi(v)} \leq C e^{-B l /\left(n^{2} \log m\right)} .
$$

Remark 4.7. The result shows that order $n^{2} \log m$ are necessary and sufficient to achieve stationarity in the $L^{2}$ or chi-square distance. The constants $A, B, C$ are independent of $m, n$ and explicitly computable. Results in Section 4.3 show that order $n^{2}$ steps are necessary and sufficient for $L^{1}$ or total variation convergence. We only know a handful of examples where the $L^{1}$ and $L^{2}$ rates differ. See, e.g., [Stong 91] or [Diaconis et al. 00].

Proof. Let $f_{k}, \lambda_{k}, 1 \leq k \leq m(n-1)$ denote the nonconstant eigenfunction, eigenvalue pairs of Proposition 4.1. By a standard identity (see, e.g., Diaconis and Saloff-Coste [Diaconis and Saloff-Coste 93]),

$$
\sum_{v^{\prime}} \frac{\left(K^{l}\left(v, v^{\prime}\right)-\pi\left(v^{\prime}\right)\right)^{2}}{\pi(v)}=\sum_{k=1}^{m(n-1)} f_{k}^{2}(v) \lambda_{k}^{2 l} .
$$

Using the known values given in Proposition 4.1, this sum equals

$$
\begin{aligned}
& \sum_{a=1}^{(n-1) / 2} 2 \cos ^{2}\left(\frac{2 \pi a j}{n}\right) \cos ^{2 l}\left(\frac{2 \pi a}{n}\right)+\sum_{a=1}^{(n-1) / 2} 2 m \sin ^{2}\left(\frac{2 \pi a j}{n}\right) \cos ^{2 l}\left(\frac{2 \pi a}{n}\right) \\
& +\sum_{a=0}^{(n-3) / 2} 2 m \frac{m-1}{m} \cos ^{2}\left(\frac{\pi a}{n}\left(\frac{n}{2}+j\right)\right) \cos ^{2 l}\left(\frac{\pi(2 a+1)}{n}\right) \text {. }
\end{aligned}
$$


In forming the last sum, we note that although the construction of the $m-1$ eigenvectors $f_{a b}$ is nonsymmetric across the $m$ petals (see Figure 15), the sum $\sum_{b=1}^{m-1} f_{a b}^{2}(i, j)$ is the same for every $i=1, \ldots, m$. In particular, for each $a$, we have

$$
\sum_{b=1}^{m-1} f_{a b}^{2}(i, j)=f_{a(m-1)}^{2}(m, j)=2 m \frac{m-1}{m} \cos ^{2}\left(\frac{\pi a}{n}\left(\frac{n}{2}+j\right)\right),
$$

which is independent of $i$ that labels the petals.

For the lower bound, discard all terms except the $a=0$ term in the final sum. The $L^{2}$ distance is bounded below by

$$
2(m-1) \cos ^{2 l}(\pi / n) .
$$

This clearly takes $l$ of order $n^{2} \log m$ to drive it to zero.

The upper bound proceeds just as for simple random walk on $n$ point circles. See [Diaconis 88, page 25] or [Saloff-Coste 04] for these classical trigonometric inequalities. Further details are omitted.

\section{3 $\quad L^{1}$ Bounds}

Let $K\left(v, v^{\prime}\right)$ be the simple random walk on the unweighted "flower" $F_{m n}$. This is reversible with unique stationary distribution $\pi(0)=1 / n, \pi(i, j)=1 /(m n)$. In this section we show that the $L^{1}$ or total variation relaxation time has order $n^{2}$, independent of $m$. Recall that

$$
\left\|K_{v}^{l}-\pi\right\|_{1}=\frac{1}{2} \sum_{v^{\prime}}\left|K^{l}\left(v, v^{\prime}\right)-\pi\left(v^{\prime}\right)\right|=\max _{S \subseteq V}\left|K^{l}(v, S)-\pi(S)\right| .
$$

Proposition 4.8. There are universal constants $A_{1}, B_{1}, B_{2}, C_{1}$ such that for every starting state $v$ and all $l$,

$$
A_{1} e^{-B_{1} l / n^{2}} \leq\left\|K_{v}^{l}-\pi\right\|_{1} \leq C_{1} e^{-B_{2} l / n^{2}} .
$$

Proof. The argument uses standard results from a random walk on an $n$-point circle. For the lower bound, the walk started at the center has vanishingly small probability of being in the top half of a petal after $\epsilon n^{2}$ steps. The walk started inside any petal has a vanishingly small chance of being in the top half of any of the other petals after $\epsilon n^{2}$ steps. In either case, there is a set $S$ with $\pi(S) \geq 1 / 10$ and $K^{l}(v, S)<1 / 10$ for $l \leq \epsilon n^{2}$ for suitable $\epsilon$ independent of $n$ or $m$. These statements combine to give the lower bound in (4.3).

For the upper bound, we construct a strong stationary time $T$ as in [Diaconis and Fill 90]. If $X_{0}, X_{1}, X_{2}, \ldots$ denotes the random walk (with $X_{0}=v$ ), then 
$T$ is a stopping time such that $P\left\{X_{T} \in S \mid T=t\right\}=\pi(S)$, for all $t$ such that $P(T=t)>0$. Such stopping times yield

$$
\left\|K_{v}^{l}-\pi\right\|_{1} \leq P(T \geq l)
$$

Let $T_{1}$ be the first hitting time of the walk started at $v$ to the state 0 . Let $T_{2}$ be a strong stationary time for the image of the walk started at 0 . An explicit construction of such a time is in Example 1 of Diaconis and Fill [Diaconis and Fill 90]. Clearly $T=T_{1}+T_{2}$ is a strong stationary time for the original walk on $F_{m n}$. Further, $T_{1}$ and $T_{2}$ are independent and

$$
P\left\{T_{i} \geq l\right\} \leq A_{i}^{\prime} e^{-B_{i}^{\prime} l / n^{2}}, \quad i=1,2
$$

for suitable constants $A_{i}^{\prime}, B_{i}^{\prime}$, by classical estimates. This proves the upper bound in (4.3).

\section{The Graph $\boldsymbol{K}_{n}-\boldsymbol{K}_{n}$}

Consider the graph $K_{n}-K_{n}$ with loops and weights as follows: vertices $(x, y)$ are end points of the extra edge with weight $A$. Edges in the left copy of $K_{n}$ with $x$ as endpoint have weight $B$. The same for vertices in the right copy of $K_{n}$ with $y$ as an endpoint. All other edges of $K_{n}$ have weight $C$. Finally every vertex different from $\{x, y\}$ has a loop with weight $D$. For $n=4$, the graph is shown in Figure 17.

Proposition 5.I. The transition matrix of the Markov chain on the edge weighted graph described above has the following set of eigenvalues:

- 1 with multiplicity one,

- $-1+\frac{A}{A+E}+\frac{F}{B+F}$ with multiplicity one,

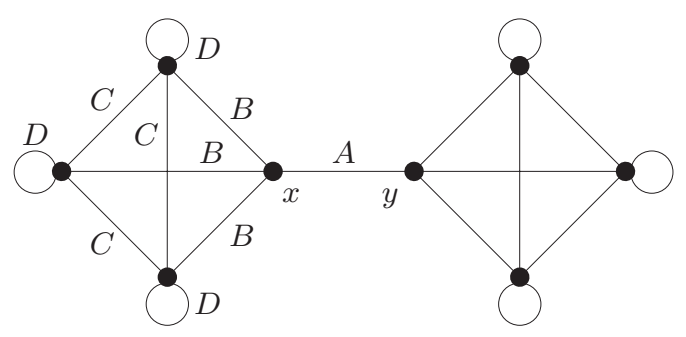

Figure 17. The graph $K_{n}-K_{n}$ with weights $A, B, C, D$. 


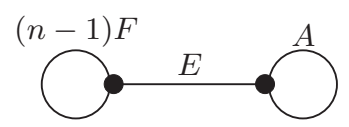

Figure 18. The orbit graph of $K_{n}-K_{n}$ under $C_{2} \ltimes\left(S_{n-1} \times S_{n-1}\right)$.

- $\frac{D-C}{B+F}$ with multiplicity $2 n-4$,

- $\frac{-A B+E F}{2(A+E)(B+F)} \pm \frac{1}{2} \sqrt{1+\frac{2 A E}{(A+E)^{2}}+\frac{2 B F}{(B+F)^{2}}+\frac{3(A F-B E)^{2}}{(A+E)^{2}(B+F)^{2}}}$ each with multiplicity one,

where $E=(n-1) B$ and $F=D+(n-2) C$.

Proof. All of our graphs have symmetry group $C_{2} \ltimes\left(S_{n-1} \times S_{n-1}\right)$ as in Section 2. From the computations of Example 1.3 in Section 2 (Corollary 2.5), the graph has at most five distinct eigenvalues, four with multiplicity one and one with multiplicity $2 n-4$. We determine the eigenvalues in the list above using a sequence of orbit graphs.

(a) The orbit graph under the full automorphism group has two states, one corresponding to the orbit $\{x, y\}$ and one corresponding to the orbit formed by the remaining $2 n-2$ points; see Figure 18 . The transition matrix of this orbit chain is

$$
\left[\begin{array}{cc}
\frac{A}{A+E} & \frac{E}{A+E} \\
\frac{E}{E+(n-1) F} & \frac{(n-1) F}{E+(n-1) F}
\end{array}\right]=\left[\begin{array}{cc}
\frac{A}{A+E} & \frac{E}{A+E} \\
\frac{B}{B+F} & \frac{F}{B+F}
\end{array}\right] .
$$

Taking traces gives the second eigenvalue shown. By Proposition 3.1 this lifts to an eigenvalue of multiplicity one for the full chain.

(b) Consider next the orbit chain under $C_{2}$ (Figure 19, left). This graph has symmetry group $S_{n-1}$ with two orbits, one of size one and the other of size $n-1$. The isotropy subgroup of the large orbit is $S_{n-2}$. The orbit chain under $S_{n-2}$ has three states (Figure 19, right) with transition matrix (only diagonals shown)

$$
\left[\begin{array}{ccc}
\frac{A}{A+(n-1) B} & * & * \\
* & \frac{D}{B+D+(n-2) C} & * \\
* & * & \frac{D+(n-3) C}{B+D+(n-2) C}
\end{array}\right]
$$

Taking traces, and using the eigenvalue found above (which also appears here), we find the third eigenvalue shown with the reported high multiplicity. Indeed, the $C_{2}$ orbit chain has three eigenvalues with multiplicity $1,1, n-2$ and the second eigenvalue has multiplicity one. 

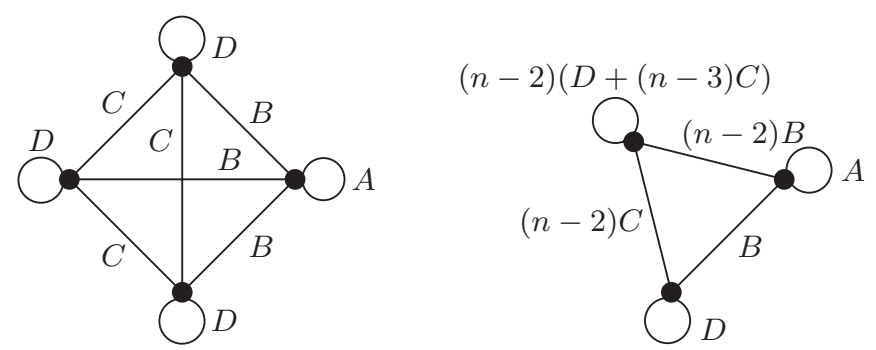

Figure 19. The orbit graphs of $K_{n}-K_{n}$ under $C_{2}$ (left) and $C_{2} \ltimes\left(S_{n-2} \times S_{n-2}\right)$ (right).

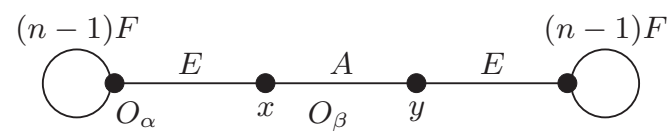

Figure 20. The orbit graph of $K_{n}-K_{n}$ under $S_{n-1} \times S_{n-1}$.

(c) To get the last two eigenvalues, consider the orbit chain for the subgroup $S_{n-1} \times S_{n-1}$. This has four orbits: $O_{\alpha}$ consisting of the $n-1$ points in the left $K_{n},\{x\},\{y\}$, and $O_{\beta}$; see Figure 20. The transition matrix is

$$
\left[\begin{array}{cccc}
\frac{F}{B+F} & \frac{B}{B+F} & 0 & 0 \\
\frac{E}{A+E} & 0 & \frac{A}{A+E} & 0 \\
0 & \frac{A}{A+E} & 0 & \frac{A}{A+E} \\
0 & 0 & \frac{B}{B+F} & \frac{F}{B+F}
\end{array}\right]
$$

This matrix has the first two displayed eigenvalues known. Solving the resulting quadratic for the last two eigenvalues gives the final result.

The bounds implicit in the following two corollaries were first derived by Mark Jerrum using quite different arguments.

Corollary 5.2. On $K_{n}-K_{n}$, for a uniform stationary distribution, the max-degree construction has $A=B=C=D=1$. The eigenvalues (in the listed order) are

$$
1,0,0, \frac{1}{2}-\frac{1}{n} \pm \frac{1}{2} \sqrt{1+\frac{4}{n}-\frac{4}{n^{2}}} .
$$

It follows that the second largest eigenvalue is $1-\frac{2}{n^{2}}+O\left(\frac{1}{n^{3}}\right)$. The Metropolis chain has slightly different weights, but the second eigenvalue is $1-\frac{c}{n^{2}}+O\left(\frac{1}{n^{3}}\right)$ for some constant $c$. 


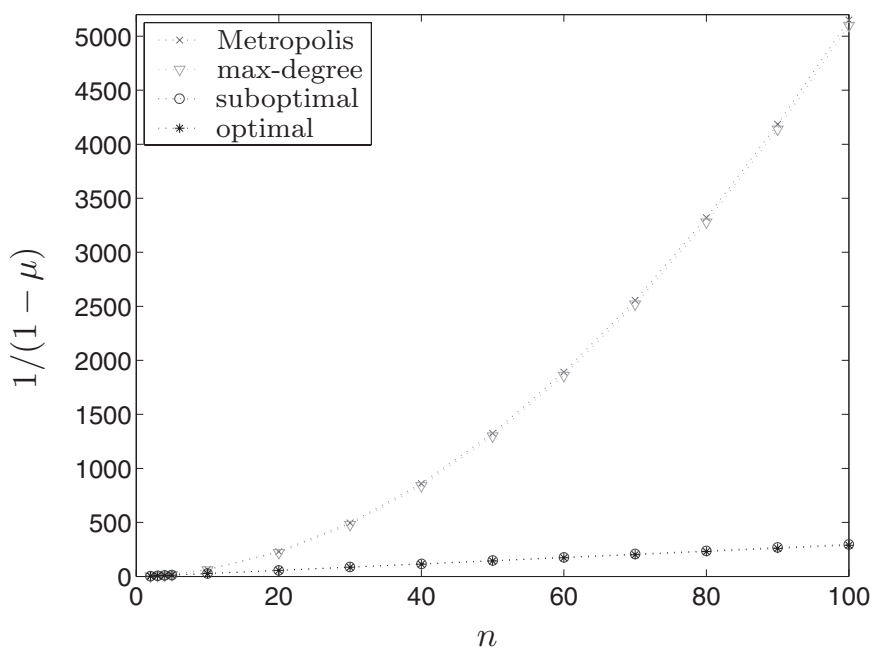

Figure $2 \mathbf{I}$. The mixing time $1 /(1-\mu)$ of $K_{n}-K_{n}$.

Corollary 5.3. A good approximation to the fastest mixing Markov chain for a uniform stationary distribution has weights $A=D=n-1$ and $B=C=1$. The eigenvalues are

$$
\begin{aligned}
& 1, \frac{1}{2}-\frac{1}{2(n-1)}, \frac{1}{2}-\frac{1}{2(n-1)}(\text { multiplicity } 2 n-4), \\
& \frac{1}{4}-\frac{1}{4(n-1)} \pm \frac{1}{4} \sqrt{9-\frac{2}{n-1}+\frac{1}{(n-1)^{2}}}
\end{aligned}
$$

We thus see that the second largest eigenvalue is $1-\frac{1}{3 n}+O\left(\frac{1}{n^{2}}\right)$.

This shows that the fastest mixing Markov chain has spectral gap at least a factor of $n$ larger than the Metropolis and max-degree chains. As argued in [Boyd et al. 04], the fastest mixing Markov chain can only improve over the Metropolis algorithm by a factor of the maximum degree of the underlying graph. Thus this is the best possible case.

Remark 5.4. Using our algorithm in [Boyd et al. 04] to find the truly fastest mixing Markov chain shows that it is slightly different than the chain of Corollary 5.3. Figure 21 shows the mixing time $1 /(1-\mu)$ (here $\mu$ is the second-largest eigenvalue magnitude) of four different Markov chains, when $n$ varies from 2 to 100 . The curve labeled as "suboptimal" is the weighted chain of Corollary 5.3; the one labeled as "optimal" is the fastest mixing Markov chain. 


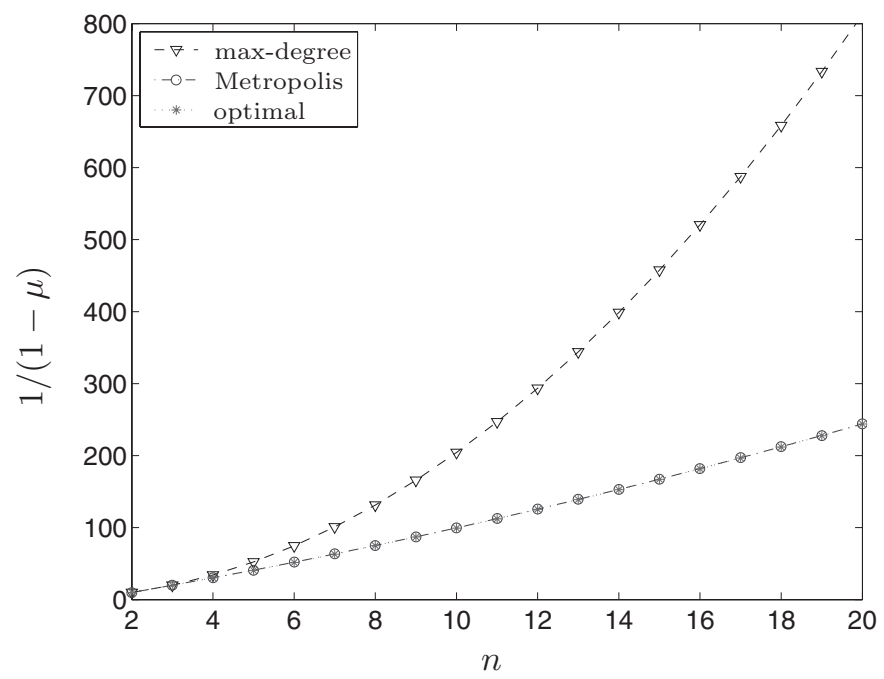

Figure 22. Mixing time $1 /(1-\mu)$ on $F_{m n}: m=10, n$ varies from 2 to 20 .

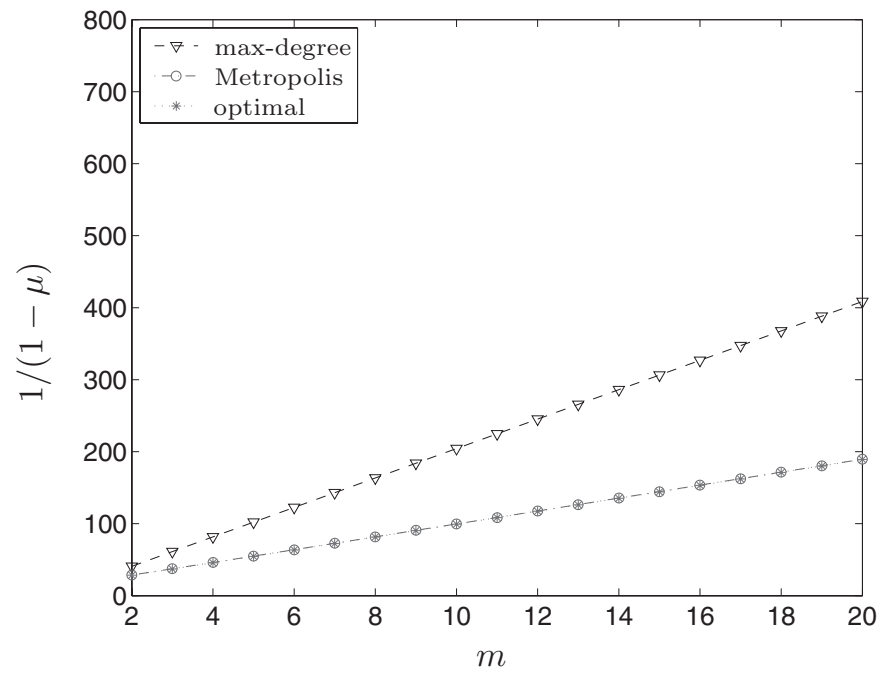

Figure 23. Mixing time $1 /(1-\mu)$ on $F_{m n}: n=10, m$ varies from 2 to 20 . 


\section{Uniform Stationary Distribution on Flowers}

In this section we give sharp bounds on the spectral gap for two different weightings of the flower graph $F_{m n}$. Both graphs have a uniform stationary distribution $\pi(v)=\frac{1}{m(n-1)+1}$. The max-degree weighting gives the chain (for $\left(v, v^{\prime}\right)$ an edge in the graph)

$$
K_{1}\left(v, v^{\prime}\right)=\frac{1}{2 m}, \quad K_{1}(v, v)=1-\frac{d_{v}}{2 m},
$$

where $d_{v}$ is the degree of vertex $v$. A picture of the weighted graph appears in Figure 3. The Metropolis weighting gives the chain (transition probabilities are symmetric on edges)

$$
\begin{aligned}
& K_{2}(0,(i, 1))=K(0,(i, n-1))=\frac{1}{2 m} \\
& K_{2}((i, j),(i, j+1))=\frac{1}{2}, \quad 1 \leq j<n-1 \\
& K_{2}((i, 1),(i, 1))=K((i, n-1),(i, n-1))=\frac{1}{2}-\frac{1}{2 m} .
\end{aligned}
$$

A picture of the weighted graph appears in Figure 2. Using symmetry analysis and geometric eigenvalue bounds, we show that the spectral gap for the maxdegree chain is a factor of $\min \{m, n\}$ times smaller than the spectral gap for the Metropolis chain. We have also found (numerically) the spectral gap for the fastest mixing Markov chain. Figure 22 shows a plot when $m=10$ and $n$ varies from 2 to 20. Figure 23 shows a plot when $n=10$ and $m$ varies from 2 to 20 . In the cases that we tried, the Metropolis and optimal chains were virtually identical.

Our numerical experiments show that the eigenvalues, as categorized in Corollary 2.2, are all distinct (as opposed to the unweighted case where there is a coalescence), and the second largest eigenvalue has multiplicity $m-1$.

\section{I The Max-Degree Chain}

Proposition 6.I. For $m \geq 2$ and odd $n \geq 3$, on the flower graph $F_{m n}$, the max-degree chain $K_{1}$ of (6.1) has second absolute eigenvalue $\mu$ satisfying

$$
1-\frac{c_{1}}{m n^{2}} \leq \mu\left(K_{1}\right) \leq 1-\frac{c_{1}^{\prime}}{m n^{2}}
$$

for universal constants $c_{1}$ and $c_{1}^{\prime}$.

Proof. Suppose throughout that $n \geq 3$ is odd; the argument for even $n$ is similar. As shown in Section 3, for any symmetric weights, all the eigenvalues appear in 


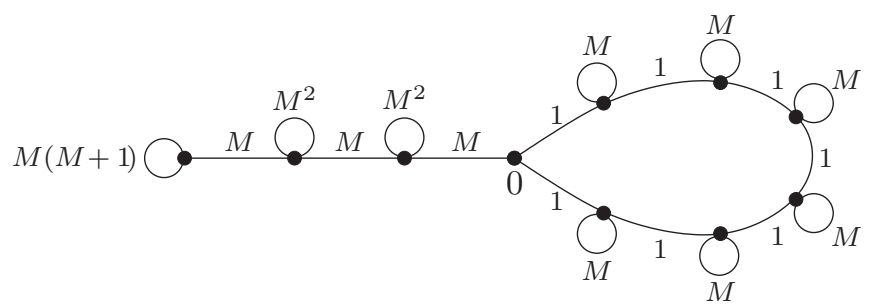

Figure 24. Orbit chain for max-degree weights on $F_{m n} ; M=2(m-1)$.

the orbit chain for the subgroup $H=S_{m-1} \ltimes C_{2}^{m-1}$. This is the random walk on the weighted "lollipop" graph shown in Figure 24 . The vertices of the $n$-cycle are labeled $0,1,2, \ldots, n-1$. The vertices of the "stem" are labeled $-1,-2, \ldots,-\frac{n-1}{2}$, from right to left. Let $|V|=m(n-1)+1$ be the number of vertices in the original graph $F_{m n}$. Then, the orbit chain has stationary distribution

$$
\begin{aligned}
& \pi_{H}(0)=\pi_{H}(i)=\frac{1}{|V|}, \quad 1 \leq i \leq n-1, \\
& \pi_{H}(i)=\frac{2(m-1)}{|V|}, \quad-\frac{n-1}{2} \leq i \leq-1 .
\end{aligned}
$$

The transition matrix for the orbit chain is

$$
\begin{aligned}
& K_{H}(0,1)=K_{H}(0, n-1)=\frac{1}{2 m}, \quad K_{H}(0,-1)=1-\frac{1}{m}, \\
& K_{H}(i, i \pm 1)=\frac{1}{2 m}, \quad K_{H}(i, i)=1-\frac{1}{m}, \quad i \neq 0,-\frac{n-1}{2}, \\
& K_{H}\left(-\frac{n-1}{2},-\frac{n-1}{2}+1\right)=\frac{1}{2 m}, \quad K_{H}\left(-\frac{n-1}{2},-\frac{n-1}{2}\right)=1-\frac{1}{2 m} .
\end{aligned}
$$

We use path arguments with Poincaré inequalities to bound the second eigenvalue $\lambda_{2}$ from above. See [Brémaud 99] for a textbook treatment; we follow the original treatment in [Diaconis and Stroock 91]. For each pair of vertices, $v \neq v^{\prime}$, there is a unique shortest path $v=v_{0}, v_{1}, \ldots, v_{h}=v^{\prime}$ with $\left(v_{i}, v_{i+1}\right)$ an edge. Loops are never used. Call this path $\gamma_{v, v^{\prime}}$ with $\left|\gamma_{v, v^{\prime}}\right|=h$. The basic Poincaré inequality says that the second eigenvalue from the top, $\lambda_{2}$, is bounded above by

$$
\lambda_{2} \leq 1-\frac{1}{A}, \quad A=\max _{e} \frac{1}{Q_{H}(e)} \sum_{\gamma_{v v^{\prime}} \ni e}\left|\gamma_{v v^{\prime}}\right| \pi_{H}(v) \pi_{H}\left(v^{\prime}\right),
$$

with the maximum over edges $e=(y, z), Q_{H}(e)=\pi_{H}(y) K_{H}(y, z)$. The sum is over paths $\gamma_{v, v^{\prime}}$ containing $e$. 
We consider two cases: edges inside the stem and edges within the cycle.

- Edges inside the stem: $e=(i, i+1),-\frac{n-1}{2} \leq i \leq-1$. Here

$$
Q_{H}(e)=\frac{2(m-1)}{|V|} \frac{1}{2 m} \text {. }
$$

Paths using $e$ start at a vertex $v$ to the left of $i$ (at most $n$ choices) and wind up at one of the points to the right of $i+1$, say $v^{\prime}$. If this point is in the stem,

$$
\pi_{H}(v) \pi_{H}\left(v^{\prime}\right)=\frac{4(m-1)^{2}}{|V|^{2}} .
$$

If the rightmost point $v^{\prime}$ is in the cycle,

$$
\pi_{H}(v) \pi_{H}\left(v^{\prime}\right)=\frac{2(m-1)}{|V|^{2}},
$$

and the number of choices for the rightmost point is at most $n$. Bound $\left|\gamma_{v, v^{\prime}}\right| \leq n$ and the number of paths by $n^{2}$, and the sum

$$
\frac{1}{Q_{H}(e)} \sum_{\gamma_{v v^{\prime}} \ni e}\left|\gamma_{v v^{\prime}}\right| \pi_{H}(v) \pi_{H}\left(v^{\prime}\right)
$$

is bounded above by

$$
\begin{aligned}
\left(\frac{2(m-1)}{2 m|V|}\right)^{-1}\left(n n^{2} \frac{4(m-1)^{2}}{|V|^{2}}+n n^{2} \frac{2(m-1)}{|V|^{2}}\right) & =\frac{m n^{3}}{|V|}(4(m-1)+2) \\
& \leq 8 m n^{2}
\end{aligned}
$$

where the last inequality used $4(m-1)+2 \leq 4 m, \frac{1}{|V|} \leq \frac{2}{m n}$. The edge $(i+1, i)$ is exactly the same.

- Edges in the cycle: $e=(i, i+1), 0 \leq i \leq n-1$. Here

$$
Q_{H}(e)=\frac{1}{|V|} \frac{1}{2 m} \text {. }
$$

Paths using $e$ may start in the stem (at most $n$ choices) and wind up in the cycle (at most $n$ choices) with

$$
\pi_{H}(v) \pi_{H}\left(v^{\prime}\right)=\frac{2(m-1)}{|V|^{2}} ;
$$

or, they may start in the cycle (at most $n$ choices) and wind up in the cycle (at most $n$ choices) with

$$
\pi_{H}(v) \pi_{H}\left(v^{\prime}\right)=\frac{1}{|V|^{2}} .
$$


Finally, they may start in the cycle (at most $n$ choices) and wind up in the stem (at most $n$ choices) with

$$
\pi_{H}(v) \pi_{H}\left(v^{\prime}\right)=\frac{2(m-1)}{|V|^{2}} .
$$

Again bounding $\left|\gamma_{v, v^{\prime}}\right| \leq n$ yields

$$
\left(\frac{1}{2 m|V|}\right)^{-1}\left(n^{3} \frac{2(m-1)}{|V|^{2}}+n^{3} \frac{1}{|V|^{2}}+n^{3} \frac{2(m-1)}{|V|^{2}}\right) \leq 12 m n^{2} .
$$

Combining the bounds, we see that $\lambda_{2} \leq 1-\frac{1}{12 m n^{2}}$.

For a lower bound on the smallest eigenvalue $\lambda_{\min }$, use Proposition 2 of [Diaconis and Stroock 91]. This requires, for each vertex $v$, a path $\sigma_{v}$ of odd length from $v$ to $v$. Here loops are allowed. For all vertices but 0 , choose the loop at $v$. At 0 , choose the path $\sigma_{0}$ as $0 \rightarrow-1 \rightarrow-1 \rightarrow 0$. The bound is

$$
\lambda_{\min } \geq-1+2 / \iota, \quad \iota=\max _{e} \frac{1}{Q_{H}(e)} \sum_{\sigma_{v} \ni e}\left|\sigma_{v}\right| \pi_{H}(v) .
$$

Bound $\left|\sigma_{v}\right| \leq 3$. The maximum occurs at the edge $(0,-1)$ and $\iota \leq 5 \mathrm{~m}$. This gives $\lambda_{\min } \geq-1+\frac{2}{5 m}$ so that $\mu\left(K_{1}\right) \leq 1-\frac{1}{12 m n^{2}}$, as stated $\left(c_{1}=\frac{1}{12}\right)$.

To prove the lower bound in Proposition 6.1, we use the variational characterization

$$
1-\lambda_{2}=\inf _{f} \frac{\mathcal{E}(f \mid f)}{\operatorname{Var}(f)}
$$

where

$$
\begin{aligned}
\mathcal{E}(f \mid f) & =\frac{1}{2} \sum_{x, y}(f(x)-f(y))^{2} \pi_{H}(x) K_{H}(x, y), \\
\operatorname{Var}(f) & =\frac{1}{2} \sum_{x, y}(f(x)-f(y))^{2} \pi_{H}(x) \pi_{H}(y) .
\end{aligned}
$$

(See, e.g., [Brémaud 99].) Thus, for any nonzero test function $f_{0}, \lambda_{2} \geq 1-$ $\mathcal{E}\left(f_{0} \mid f_{0}\right) / \operatorname{Var}\left(f_{0}\right)$. Choose $f_{0}$ to vanish on the stem, and on the cycle define

$$
f_{0}(j)= \begin{cases}j, & 0 \leq j \leq \frac{n-1}{4} \\ \frac{n-1}{2}-j, & \frac{n-1}{4}<j \leq \frac{n-1}{2} \\ -f_{0}(n-j), & -\frac{n+1}{2} \leq j \leq n-1 .\end{cases}
$$

Then, under $\pi_{H}, f_{0}$ has mean zero, $\operatorname{Var}\left(f_{0}\right)$ asympotic to $\frac{1}{|V|} B n^{3}$, and $\mathcal{E}\left(f_{0} \mid f_{0}\right)$ asymptotic to $B^{\prime} \frac{n}{m|V|}$, for computible constants $B$ and $B^{\prime}$. Thus, for explicit constant $c_{1}^{\prime}, \mathcal{E}\left(f_{0} \mid f_{0}\right) / \operatorname{Var}\left(f_{0}\right) \leq \frac{c_{1}^{\prime}}{m n^{2}}$. This proves the lower bound and so completes the proof of Proposition 6.1. 


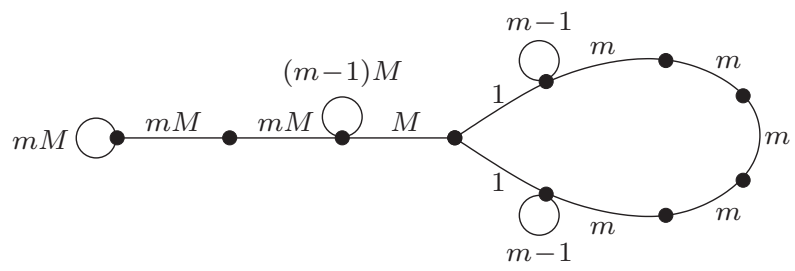

Figure 25. Orbit chain for Metropolis weights on $F_{m n} ; M=2(m-1)$.

\subsection{The Metropolis Chain}

Proposition 6.2. For $m \geq 2$ and odd $n \geq 3$, on the flower graph $F_{m n}$, the Metropolis chain $K_{2}$ of (6.2) has second absolute eigenvalue $\mu$ satisfying

$$
1-c_{2} \frac{(m+n)}{m n^{2}} \leq \mu\left(K_{2}\right) \leq 1-c_{2}^{\prime} \frac{1}{(m+n) n}
$$

with $c_{2}, c_{2}^{\prime}$ universal constants.

Proof. As for Proposition 6.1, all the eigenvalues of $K_{2}$ appear in the orbit chain for the subgroup $H=S_{m-1} \ltimes C_{2}^{m-1}$. This is the random walk on the weighted "lollipop" graph shown in Figure 25. The vertices of are labeled $0,1,2, \ldots, n-1$ on the $n$-cycle, and $-1,-2, \ldots,-\frac{n-1}{2}$ on the "stem" from right to left. As in Section 6.1 , let $|V|=m(n-1)+1$. The stationary distribution is

$$
\begin{aligned}
& \pi_{H}(0)=\pi_{H}(i)=\frac{1}{|V|}, \quad 1 \leq i \leq n-1, \\
& \pi_{H}(i)=\frac{2(m-1)}{|V|}, \quad-\frac{n-1}{2} \leq i \leq-1 .
\end{aligned}
$$

The transition matrix for the orbit chain is

$$
\begin{aligned}
& K_{H}(0,1)=K_{H}(0, n-1)=\frac{1}{2 m}, \quad K_{H}(0,-1)=1-\frac{1}{m}, \\
& K_{H}(1,0)=K(n-1,0)=K_{H}(-1,0)=\frac{1}{2 m}, \\
& K_{H}(1,1)=K(n-1, n-1)=K_{H}(-1,-1)=\frac{1}{2}-\frac{1}{2 m}, \\
& K_{H}(i, i+1)=K_{H}(i+1, i)=\frac{1}{2}, \quad i \neq-1,0, n-1, \\
& K_{H}\left(-\frac{n-1}{2},-\frac{n-1}{2}\right)=\frac{1}{2} .
\end{aligned}
$$


Again, there is a unique shortest path $\gamma_{v, v^{\prime}}$ between any two vertices $v$ to $v^{\prime}$. Using the Poincaré inequality (6.3) for $\lambda_{2}$ on the Metropolis chain yields an upper bound $1-\frac{c}{m n^{2}}$ for some constant $c$. This has the same order as the maximum-degree chain and is unsatisfactory. Instead, we use another form of Poincaré inequality derived in Proposition 1 of [Diaconis and Stroock 91]:

$$
\lambda_{2} \leq 1-\frac{1}{\kappa}, \quad \kappa=\max _{e} \sum_{\gamma_{v v^{\prime}} \ni e}\left|\gamma_{v v^{\prime}}\right|_{Q_{H}} \pi_{H}(v) \pi_{H}\left(v^{\prime}\right),
$$

where

$$
\left|\gamma_{v v^{\prime}}\right|_{Q_{H}}=\sum_{e \in \gamma_{v v^{\prime}}} Q_{H}(e)^{-1}
$$

First, we bound $\left|\gamma_{v v^{\prime}}\right|_{Q_{H}}$ for all possible paths. There are three cases:

- Paths in the stem: $-\frac{n-1}{2} \leq v, v^{\prime} \leq-1$. Edges in such paths have $Q_{H}(e)=$ $\frac{2(m-1)}{|V|} \frac{1}{2}$, and we bound the number of edges in a path by $n$. Therefore,

$$
\left|\gamma_{v v^{\prime}}\right|_{Q_{H}} \leq n\left(\frac{2(m-1)}{|V|} \frac{1}{2}\right)^{-1}=\frac{n|V|}{m-1} \triangleq\left|\gamma^{(-)}\right| .
$$

- Paths in the cycle: $0 \leq v, v^{\prime} \leq n-1$. The two edges incident to 0 have $Q_{H}(e)=\frac{1}{|V|} \frac{1}{2 m}$, and other edges have $Q_{H}(e)=\frac{1}{|V|} \frac{1}{2}$;

$$
\left|\gamma_{v v^{\prime}}\right|_{Q_{H}} \leq 2\left(\frac{1}{|V|} \frac{1}{2 m}\right)^{-1}+n\left(\frac{1}{|V|} \frac{1}{2}\right)^{-1}=(4 m+2 n)|V| \triangleq\left|\gamma^{(+)}\right| .
$$

- Paths across the center: $-\frac{n-1}{2} \leq v \leq-1,0 \leq v^{\prime} \leq n-1$. The edge $(-1,0)$ has $Q_{H}(e)=\frac{2(m-1)}{|V|} \frac{1}{2 m}$. Contribution from other edges are bounded by $\left|\gamma^{(-)}\right|$and $\left|\gamma^{(+)}\right|$;

$$
\left|\gamma_{v v^{\prime}}\right|_{Q_{H}} \leq\left|\gamma^{(-)}\right|+\left|\gamma^{(+)}\right|+\left(\frac{2(m-1)}{2 m|V|}\right)^{-1} \leq(5 m+3 n)|V| \triangleq\left|\gamma^{( \pm)}\right| .
$$

Now, we bound the quantities $\kappa(e) \triangleq \sum_{\gamma_{v v^{\prime}} \ni e}\left|\gamma_{v v^{\prime}}\right|_{Q_{H}} \pi_{H}(v) \pi_{H}\left(v^{\prime}\right)$ for all the edges. There are three cases:

- Edges in the stem: $e=(i, i+1),-\frac{n-1}{2} \leq i \leq-2$. Paths using $e$ start at a vertex $v$ to the left of $i$, and wind up at a vertex $v^{\prime}$ to the right of $i+1$. If $v^{\prime}$ is in the stem, $\pi_{H}(v) \pi_{H}\left(v^{\prime}\right)=\frac{4(m-1)^{2}}{|V|^{2}}$ and $\left|\gamma_{v v^{\prime}}\right|_{Q_{H}} \leq\left|\gamma^{(-)}\right|$. If $v^{\prime}$ is in the cycle, $\pi_{H}(v) \pi_{H}\left(v^{\prime}\right)=\frac{2(m-1)}{|V|^{2}}$ and $\left|\gamma_{v v^{\prime}}\right|_{Q_{H}} \leq\left|\gamma^{( \pm)}\right|$. We bound the number of paths containing $e$ by $n^{2}$ in either case;

$$
\kappa(e) \leq n^{2}\left|\gamma^{(-)}\right| \frac{4(m-1)^{2}}{|V|^{2}}+n^{2}\left|\gamma^{( \pm)}\right| \frac{2(m-1)}{|V|^{2}} \leq 20(m+n) n .
$$


- The edge $(-1,0)$. Paths using $e$ start in the stem and end in the cycle, so $\pi_{H}(v) \pi_{H}\left(v^{\prime}\right)=\frac{2(m-1)}{|V|^{2}}$ and $\left|\gamma_{v v^{\prime}}\right|_{Q_{H}} \leq\left|\gamma^{( \pm)}\right|$. We bound the number of paths containing $e$ by $n^{2}$;

$$
\kappa(e) \leq n^{2}\left|\gamma^{( \pm)}\right| \frac{2(m-1)}{|V|^{2}} \leq(20 m+12 n) n .
$$

- Edges in the cycle $e=(i, i+1), 0 \leq i \leq n-1$. Paths using $e$ may start in the stem and end in the cycle (analysis is same as previous case), or start in the cycle and end in the cycle. In the second case, $\pi_{H}(v) \pi_{H}\left(v^{\prime}\right)=\frac{1}{|V|^{2}}$ and $\left|\gamma_{v v^{\prime}}\right|_{Q_{H}} \leq\left|\gamma^{(+)}\right|$

$$
\kappa(e) \leq n^{2}\left|\gamma^{( \pm)}\right| \frac{2(m-1)}{|V|^{2}}+n^{2}\left|\gamma^{(+)}\right| \frac{1}{|V|^{2}} \leq 20(m+n) n
$$

All the $\kappa(e)$ are bounded above by $20(m+n) n$. This shows that $\lambda_{2} \leq 1-\frac{1}{20(m+n) n}$ (here $c_{2}=\frac{1}{20}$ ). Again, paths of odd length can be used to show $\mu=\lambda_{2}$.

To prove the lower bound, use the variational characterization (6.4) and the following test function $f_{0}$. Choose $f_{0}$ to be zero on the stem and the center 0 . On the cycle, define $f_{0}(j)=j-\frac{n}{2}$ for $1 \leq j \leq n-1$. Then, under $\pi_{H}, f_{0}$ has mean zero and variance asymptotic to $\frac{1}{|V|} B n^{3}$ for a computible constant $B$. Next,

$$
\mathcal{E}\left(f_{0} \mid f_{0}\right)=2\left(0-\left(1-\frac{n}{2}\right)\right)^{2} \frac{1}{|V|} \frac{1}{2 m}+(n-2) 1^{2} \frac{1}{|V|} \frac{1}{2},
$$

where the first term comes from the two edges incident to the center 0 and the second term comes from the rest $n-2$ edges. It can be seen that $\mathcal{E}\left(f_{0} \mid f_{0}\right)$ has asymptotic order of $\frac{(m+n) n}{m|V|}$. Thus, for some explicit constant $c_{2}^{\prime}, \mathcal{E}\left(f_{0} \mid f_{0}\right) / \operatorname{Var}\left(f_{0}\right) \leq$ $c_{2}^{\prime} \frac{m+n}{m n^{2}}$, and this gives the claimed lower bound on $\lambda_{2}$.

Remark 6.3. For $m=O(n)$, the upper and lower bounds have the same order, $\mu\left(K_{2}\right) \sim 1-\frac{c_{3}}{n^{2}}$. For $m=o(n), 1-\frac{c_{4}}{m n} \leq \mu \leq 1-\frac{c_{4}^{\prime}}{n^{2}}$. For $n=o(m), 1-\frac{c_{5}}{n^{2}} \leq$ $\mu \leq 1-\frac{c_{5}^{\prime}}{m n}$. Here, $c_{i}, c_{i}^{\prime}$ are computable universal constants. Compared with Proposition 6.1, the spectral gap for the Metropolis chan is a factor of $\min \{m, n\}$ times larger than the spectral gap for the max-degree chain.

Acknowledgments. We thank Daniel Bump, Robin Forman, Mark Jerrum, Arun Ram, and Andrez Zuk for incisive contributions to this paper. 


\section{References}

[Aldous and Fill 03] D. Aldous and J. Fill. Reversible Markov Chains and Random Walks on Graphs. Preprint available from World Wide Web (http://statwww.berkeley.edu/users/aldous/RWG/book.html). Manuscript, 2003.

[Babai 95] L. Babai. "Automorphism Groups, Isomorphism, Reconstruction." In Handbook of Combinatorics, Volume 2, edited by R. Graham et al., pp. 14471540. Amsterdam: Elsevier, 1995.

[Belsley 98] E. Belsley. "Rates of Convergence of Random Walk on Distance-Regular Graphs." Probability Theory and Related Fields 112:4 (1998), 493-533.

[Boyd et al. 04] S. Boyd, P. Diaconis, and L. Xiao. "Fastest Mixing Markov Chain on a Graph." SIAM Review 46 (2004), 667-689.

[Brémaud 99] P. Brémaud. Markov Chains, Gibbs Fields, Monte Carlo Simulation and Queues, Texts in Applied Mathematics 31. Berlin-Heidelberg: Springer-Verlag, 1999.

[Cameron 99] P. Cameron. Permutation Groups. Cambridge, UK: Cambridge University Press, 1999.

[Cameron 03] P. Cameron. "Coherent Configurations, Association Schemes and Permutation Groups." In Groups, Combinatorics and Geometry, edited by A. Ivanov, M. Liebeck, and J. Saxl, pp. 55-72. River Edge, NJ: World Scientific, 2003.

[Chan and Godsil 97] A. Chan and C. Godsil. "Symmetry and Eigenvectors." In Graph Symmetry, Algebraic Methods and Applications, edited by G. Hahn and G. Sabidussi, pp. 75-106. Dordrecht, The Netherlands: Kluwer, 1997.

[Chung 97] F. R. K. Chung. Spectral Graph Theory. Providence, RI: American Mathematics Society, 1997.

[Curtis and Reiner 62] C. Curtis and I. Reiner. Representation Theory of Finite Groups and Associative Algebras. New York: Wiley, 1962.

[Cvetković et al. 95] D. Cvetković, M. Doob, and H. Sachs. Spectra of Graphs, Third edition. New York: Academic Press, 1995.

[Diaconis 88] P. Diaconis. Group Representations in Probability and Statistics. Hayward, CA: IMS, 1988.

[Diaconis and Fill 90] P. Diaconis and J. A. Fill. "Strong Stationary Times via a New Form of Duality." The Annals of Probability 18:4 (1990), 1483-1522.

[Diaconis et al. 00] P. Diaconis, S. Holmes, and R. M. Neal. "Analysis of a Nonreversible Markov Chain Sampler." The Annals of Applied Probability 10 (2000), $726-752$.

[Diaconis and Saloff-Coste 93] P. Diaconis and L. Saloff-Coste. "Comparison Theorems for Reversible Markov Chains." The Annals of Applied Probability 3 (1993), 696730 .

[Diaconis and Stroock 91] P. Diaconis and D. Stroock. "Geometric Bounds for Eigenvalues of Markov Chains." The Annals of Applied Probability 1:1 (1991), 36-61. 
[Dieudonne 78] J. Dieudonne. Treatise on Analysis VI. New York: Academic Press, 1978.

[Erdös et al. 66] P. Erdös, A. Renyi, and V. Sós. "On a Problem of Graph Theory." Studia Sci. Math. Hungar. 1 (1966), 215-235.

[Fässler and Stiefel 92] A. Fässler and E. Stiefel. Group Theoretical Methods and Their Applications. Boston: Birkhäuser, 1992.

[Feller 68] W. Feller. An Introduction to Probability Theory and Its Applications, Vol. 1. New York: Wiley, 1968.

[Gatermann and Parrilo 04] K. Gatermann and P. A. Parrilo. "Symmetry Groups, Semidefinite Programs, and Sums of Squares." Journal of Pure and Appl. Algebra 192:1-3 (2004), 95-128.

[Godsil and Royle 01] C. Godsil and G. Royle. Algebraic Graph Theory. New York: Springer, 2001.

[Halverson and Ram 96] T. Halverson and A. Ram. "Murnaghan-Nakayama Rules for Characters of Iwahori-Hecke Algebras of Classical Type." Trans. of Amer. Math. Soc. 348 (1996), 3967-3995.

[James and Kerber 81] G. James and A. Kerber. The Representation Theory of the Symmetric Group. Reading, MA: Addison-Wesley, 1981.

[Kac 47] M. Kac. "Random Walk and the Theory of Brownian Motion." American Mathematical Monthly 54 (1947), 369-391.

[Kemeney and Snell 60] J. G. Kemeney and J. L. Snell. Finite Markov Chains. Princeton, NJ: Van Nostrand, 1960.

[Lauri and Scapellato 03] J. Lauri and R. Scapellato. Topics in Graph Automorphisms and Reconstruction. Cambridge, UK: Cambridge University Press, 2003.

[Liu 01] J. Liu. Monte Carlo Strategies in Scientific Computing, Springer Series in Statistics XVI. New York: Springer-Verlag, 2001.

[Mackey 78] G. Mackey. Unitary Group Representations in Physics, Probability and Number Theory. New York: Benjamin, 1978.

[Neumann 79] P. Neumann. "A Lemma that is not Burnsides." Math. Scientist 4 (1979), 13-141.

[Parrilo et al. 04] P. A. Parrilo, L. Xiao, S. Boyd, and P. Diaconis. "Fastest Mixing Markov Chain on Graphs with Symmetries." Manuscript in preparation, 2004.

[Pinsky 80] M. Pinsky. "The Eigenvalues of an Equilateral Triangle." SIAM Journal on Mathematical Analysis 11 (1980), 819-827.

[Pinsky 85] M. Pinsky. "Completeness of the Eigenfunctions of the Equilateral Triangle." SIAM Journal on Mathematical Analysis 16 (1985), 848-851.

[Saloff-Coste 97] L. Saloff-Coste. "Lectures on Finite Markov Chains." In Lectures on Probability Theory and Statistics, Lecture Notes in Mathematics 1665, edited by P. Bernard, pp. 301-413. Berlin: Springer-Verlag, 1997.

[Saloff-Coste 04] L. Saloff-Coste. "Random Walk on Finite Groups." In Probability on Discrete Structures, Encyclopedia of Mathematical Sciences 110, edited by H. Kesten, pp. 263-346. Berlin: Springer-Verlag, 2004 
[Sternberg 94] S. Sternberg. Group Theory and Physics. Cambridge, UK: Cambridge University Press, 1994.

[Stong 91] R. Stong. "Choosing a Random Spanning Tree: A Case Study." Theoretical Probab. 4 (1991), 753-766.

Stephen Boyd, Department of Electrical Engineering, Stanford University, Stanford, CA 94305 (boyd@stanford.edu)

Persi Diaconis, Department of Statistics and Department of Mathematics, Stanford University, Stanford, CA 94305

Pablo Parrilo, Laboratory for Information and Decision Systems, Massachusetts Institute of Technology, Cambridge, MA 02139-4307 (parrilo@mit.edu)

Lin Xiao, Center for the Mathematics of Information, California Institute of Technology, Pasadena, CA 91125-9300 (lxiao@caltech.edu)

Received December 6, 2003; accepted May 21, 2004. 